\title{
Parametric decay instabilities near the second-harmonic upper hybrid resonance in
} fusion plasmas

Paper

Hansen, S. K.; Nielsen, S. K.; Stober, J.; Rasmussen, J.; Stejner, M.; Hoelzl, M.; Jensen, T.

Published in:

Nuclear Fusion

Link to article, DOI:

10.1088/1741-4326/aba802

Publication date:

2020

Document Version

Peer reviewed version

Link back to DTU Orbit

Citation (APA):

Hansen, S. K., Nielsen, S. K., Stober, J., Rasmussen, J., Stejner, M., Hoelzl, M., \& Jensen, T. (2020).

Parametric decay instabilities near the second-harmonic upper hybrid resonance in fusion plasmas: Paper. Nuclear Fusion, 60(10), [106008]. https://doi.org/10.1088/1741-4326/aba802

\section{General rights}

Copyright and moral rights for the publications made accessible in the public portal are retained by the authors and/or other copyright owners and it is a condition of accessing publications that users recognise and abide by the legal requirements associated with these rights.

- Users may download and print one copy of any publication from the public portal for the purpose of private study or research.

- You may not further distribute the material or use it for any profit-making activity or commercial gain

- You may freely distribute the URL identifying the publication in the public portal 


\title{
Parametric Decay Instabilities near the Second-Harmonic Upper Hybrid Resonance in Fusion Plasmas
}

\author{
S K Hansen ${ }^{1}$, S K Nielsen ${ }^{2}$, J Stober ${ }^{3}$, J Rasmussen ${ }^{2}$, M \\ Stejner $^{2}$, M Hoelzl ${ }^{3}$, T Jensen ${ }^{2}$, and the ASDEX Upgrade \\ Team $^{4}$ \\ ${ }^{1}$ Plasma Science and Fusion Center, Massachusetts Institute of Technology, \\ Cambridge, Massachusetts 02139, United States of America \\ 2 Department of Physics, Technical University of Denmark, DK-2800 Kgs. Lyngby, \\ Denmark \\ ${ }^{3}$ Max-Planck-Institut für Plasmaphysik, D-85748 Garching b. München, Germany \\ ${ }^{4}$ See Author List in Meyer H for the ASDEX Upgrade Team 2019 Nucl. Fusion 59 \\ 112014 \\ E-mail: soerenkh@psfc.mit.edu
}

\begin{abstract}
Parametric decay instabilities (PDIs) lead to the generation of strong frequency-shifted radiation when powerful X-mode polarized microwaves, injected for electron cyclotron resonance heating (ECRH) of fusion plasmas, cross a region of nonmonotonic electron density near the second-harmonic upper hybrid resonance (UHR). For the standard second-harmonic X-mode ECRH scenarios used at the ASDEX Upgrade tokamak, the second-harmonic UHR occurs near the plasma edge, meaning that PDIs occur in connection with phenomena leading to non-monotonic edge electron density profiles, e.g., blobs, edge-localized modes (ELMs), and inter-ELM modes. We present the first study of strong signals near half the ECRH frequency in fusion-relevant plasmas and demonstrate their PDI-like features, such as an ECRH power threshold required for their occurrence, through analog modulations of the ECRH power. The signals near half the ECRH frequency are found to be far more prevalent than expected based on previous theories, and we hence present a qualitative explanation of their origin, in terms of cross-polarized microwaves generated through nonlinear interactions of the waves involved in the PDIs, which captures the basic features of the experimental observations. We additionally present examples of the PDI-related microwave signals just below the ECRH frequency which indicate the location of the microwave source. Furthermore, based on a nonlinear magnetohydrodynamics simulation of an ELM crash in ASDEX Upgrade, performed using the JOREK code, the duration of the PDI-like microwave spikes observed in connection with ELMs is shown to match the transit time of an ELM filament though an ECRH beam. Finally, the PDI power threshold expected theoretically, calculated using the electron density and temperature profiles from the JOREK simulation, is shown to match the experimentally observed values.
\end{abstract}

Keywords: parametric decay instability, ECRH, upper hybrid waves, wave trapping, microwave diagnostics, ELMs, blobs 


\section{Introduction}

Parametric decay instabilities (PDIs) are nonlinear phenomena in which a largeamplitude pump wave decays to two daughter waves once its amplitude exceeds a threshold determined by the coupling strength between the three waves. Due to energy and momentum conservation, the frequencies and wave vectors of the daughter waves should sum to those of the pump wave. The wave vector selection rule particularly means that PDIs will only occur in a narrow region in inhomogeneous plasmas [1 6 , ordinarily increasing the PDI power thresholds of electron cyclotron resonance heating (ECRH) beams in fusion-relevant plasmas to $\gtrsim 10 \mathrm{MW}$ [4,5], which is an order of magnitude above the power available from the gyrotron sources $(\sim 1 \mathrm{MW})$ typically used for ECRH in such plasmas [7,8]. However, the PDI power thresholds may be reduced to a level accessible with ECRH when the daughter waves excited by the PDIs are trapped, since the amplification region is traversed multiple times in such cases $[9]$. This can particularly happen if the upper hybrid (UH) frequency has a maximum slightly exceeding half the ECRH frequency, which allows decay of the electromagnetic (X-mode) ECRH wave to two, approximately half-frequency, UH plasmons trapped around the maximum [5, 6, 10 15]. Such PDIs have previously been reported in lowtemperature plasma filaments [16, 17], during rotating magnetic islands at the TEXTOR tokamak [18, 19], in connection with edge-localized modes (ELMs) at the ASDEX Upgrade tokamak [6, 20,21], and have recently been shown to occur in particle-in-cell simulations as well [22]. However, the experimental investigations performed under fusion-relevant conditions so far have only focused on microwave signals close to the ECRH frequency, which orginate from combination of the main daughter waves near half the ECRH frequency [10], limiting their usefulness for exploring the basic PDI mechanism. Here, we present the first investigation of microwave signals near half the ECRH frequency under fusion-relevant conditions and conclusively demonstrate the validity of the theoretical framework.

The strong, frequency-shifted radiation generated by the above PDIs poses a threat to microwave diagnostics such as electron cyclotron emission (ECE) in connection with magnetic islands [6]. In these cases the PDIs may even lead to the conversion of a very large fraction (up to $80 \%$ ) of the ECRH power to UH plasmon power according to current theories 14.15], changing the power deposition and current drive characteristics; PDI-mediated absorption of $45 \%$ of the ECRH power has been demonstrated in lowtemperature plasma filament experiments [17]. On the other hand, as shown in the present paper, the PDIs allow detection of structures possessing non-monotonic electron density profiles along an ECRH beam near the edge of fusion plasmas, e.g., ELM filaments, inter-ELM modes [23], and blobs [24], since the wave amplitudes generated in these cases are generally insufficient to damage microwave diagnostics. The question of the ECRH power fraction converted to waves near half the ECRH frequency in the above structures is not addressed in detail, owing to the difficulty of measuring the exact ECRH heating efficiency or trapped wave amplitude, but we do present calibrated 
measurements of the microwave spectral power density near the ECRH frequency and half the ECRH frequency observed on the low-field side of the plasma, which should facilitate such investigations.

As microwave bursts in connection with ELMs have been observed under a number of different experimental conditions in the past [6, 20, 21, 25, 33], we provide a brief overview of these bursts and their connection, or lack thereof, to PDIs. Many of the earlier observations of microwave bursts during ELMs occurred without ECRH [33] and additionally had frequencies proportional to the magnetic field strength [29, 33]. This is a strong indication that they do not originate from PDIs, which require an ECRH beam to drive the instabilities and lead to emissions with frequencies depending on the ECRH frequency, but rather from ECE associated with fast electrons. Several mechanisms for ECE bursts during ELMs exist, which may describe the observations for different experimental conditions. In larger devices, such as the JET and TFTR tokamaks, the bursts have been explained by a reduction of the optical thickness of the plasma edge during ELMs, allowing ECE from fast electrons in the core to escape the plasma [25 28]. In medium-sized devices, such as the DIII-D, MAST, and ASDEX Upgrade tokamaks, the bursts have on the other hand been explained by ECE from fast electrons near the plasma edge generated during the ELM crash [29,31-33]. In contrast to the above observations, the microwave bursts from ASDEX Upgrade discussed in this paper are shown to require the ECRH beam power to exceed a certain threshold, to be strongest for receivers viewing an ECRH beam near the plasma edge, and to have frequencies confined to narrow bands around the ECRH frequency and half the ECRH frequency, which are all features consistent with a PDI-origin; additionally, similar signals are found in connection with inter-ELM modes and blobs. We do, however, note that previous observations of microwave bursts in connection with ELMs during collective Thomson scattering (CTS) experiments at ASDEX Upgrade [30] may be consistent with ECE from fast electrons generated by ECRH of the ELM filaments themselves, as the fundamental and second-harmonic electron cyclotron resonances (ECRs) occur in the high- and low-field side scrape-off layers, respectively, in these experiments.

This paper is organized as follows. Section 2 presents the theory of PDIs near the second-harmonic upper hybrid resonance (UHR) used to interpret the experimental results. Section 3 describes the experimental setup used to detect PDIs at ASDEX Upgrade. Section 4 presents the experimental results demonstrating the occurrence of PDIs near the plasma edge at ASDEX Upgrade; it further shows that PDIs near the plasma edge are expected in connection with an ELM at ASDEX Upgrade, simulated using the nonlinear magnetohydrodynamics (MHD) code JOREK 34 36]. Section 5 discusses the interpretation of the obtained results. Finally, section 6 presents our conclusions. 


\section{Theory of PDIs near the Second-Harmonic UHR}

The primary PDI near the second-harmonic UHR involves decay of an electromagnetic $\mathrm{X}$-mode pump wave to two UH plasmons. For propagation perpendicular to the magnetic field, $\mathbf{B}=B \mathbf{e}_{z}$, the linear $\mathrm{UH}$ plasmon dispersion relation is given by $[6]$

$\mathcal{D}=\mathcal{D}^{\prime}+\mathrm{i} \mathcal{D}^{\prime \prime}=0, \quad \mathcal{D}^{\prime}=\ell_{T e}^{2} k^{4}+S k^{2}-\frac{\omega^{2}}{c^{2}}\left(S^{2}-D^{2}\right), \quad \mathcal{D}^{\prime \prime}=k^{2} \frac{\omega_{p e}^{2}\left(\omega^{2}+\omega_{c e}^{2}\right)}{\left(\omega^{2}-\omega_{c e}^{2}\right)^{2}} \frac{\nu_{e}}{\omega}$,

where

$$
\ell_{T e}^{2}=\frac{3 \omega_{p e}^{2}\left(T_{e} / m_{e}\right)}{\left(4 \omega_{c e}^{2}-\omega^{2}\right)\left(\omega^{2}-\omega_{c e}^{2}\right)}, \quad S=1-\frac{\omega_{p e}^{2}}{\omega^{2}-\omega_{c e}^{2}}, \quad D=\frac{\omega_{c e}}{\omega} \frac{\omega_{p e}^{2}}{\omega^{2}-\omega_{c e}^{2}},
$$

with $\omega$ being the (complex) frequency, $k$ being the wave number, $\omega_{p e}=\sqrt{e^{2} n_{e} /\left(\epsilon_{0} m_{e}\right)}$ being the electron plasma frequency, $\omega_{c e}=-e B / m_{e}$ being the electron cyclotron frequency, $\nu_{e}$ being the electron Krook collision frequency (generally set equal to the electron-ion Coulomb collision frequency [37]), $T_{e}$ being the electron temperature in energy units, $n_{e}$ being the electron density, $m_{e}$ being the electron mass, $e$ being the elementary charge, $\epsilon_{0}$ being the vacuum permittivity, and $c$ being the vacuum speed of light. In the weakly collisional regime relevant to fusion plasmas, $\nu_{e} /|\omega| \ll 1$, $\mathcal{D}^{\prime \prime}$ may be considered a perturbation, such that the zeroth order solution of (1) becomes $\mathcal{D}_{j}^{\prime}=0$, where $\mathcal{D}_{j}^{\prime}$ is $\mathcal{D}^{\prime}$ evaluated at the real frequency $\left(\omega=\omega_{j}\right)$ and wave number $\left(k=k_{j}\right)$. $\mathcal{D}_{j}^{\prime}=0$ is a quadratic equation for $k_{j}^{2}$, which may be solved to give

$$
\left(k_{j x}^{ \pm}\right)^{2}=-\frac{S_{j}}{2 \ell_{T e j}^{2}}\left(1 \pm \sqrt{1+\frac{4 \ell_{T e j}^{2} \omega_{j}^{2}}{c^{2}} \frac{S_{j}^{2}-D_{j}^{2}}{S_{j}^{2}}}\right)-k_{j y}^{2},
$$

where we have used that $k_{j}^{2}=k_{j x}^{2}+k_{j y}^{2}$, since $\mathbf{k} \perp \mathbf{B} \| z$, and written $S_{j}, D_{j}, \ell_{T e j}$ for $S, D, \ell_{T e}$ evaluated at $\omega=\omega_{j}$. The "+" and "-" roots of (3) coincide when $S_{j}^{2}=-4\left(\ell_{T e j}^{2} \omega_{j}^{2} / c^{2}\right)\left(S_{j}^{2}-D_{j}^{2}\right)$, which is known as the warm UHR; when $T_{e} \ll m_{e} c^{2}$, this condition becomes $S_{j} \approx 0$, i.e., the well-known cold UHR at which $\omega_{j}=\sqrt{\omega_{p e}^{2}+\omega_{c e}^{2}}=$ $\omega_{U H}$, with $\omega_{U H}$ being the UH frequency. Far from the UHR, the "+ "root of (3) corresponds to electrostatic electron Bernstein waves (EBWs), while the "-" root corresponds to electromagnetic X-mode waves [6]; X-mode waves reaching the UHR will be converted to EBWs and vice versa [38 41]. Cold X-mode waves are propagating for $\omega_{j}<\omega_{U H}$, which will also be the case for EBWs, provided that $\omega_{j}<2\left|\omega_{c e}\right|[6]$. Under these circumstances, a pair of UH plasmons from the EBW and X-mode branches can be trapped around a maximum of $\omega_{U H}$ if points where $\omega_{j}=\omega_{U H}$ exist on both sides of the maximum. To illustrate this, we consider a slab with plasma parameters only varying along the $x$ direction (and $\mathbf{B} \| z$ ) in pane (a) of figure 1; only the waves with $j=1$ should be considered for the time being. In such a system, $B$ is constant and maxima of $\omega_{U H}$ coincide with maxima of $n_{e}$. If UHRs (values of $n_{e}$ leading to $\omega_{1}=\omega_{U H}$ ) are found on both sides of the maximum of $n_{e}$, the UH plasmons will be trapped around the maximum, where they are converted from EBWs to X-mode waves, and vice versa, every time they reach a UHR; the $x$ values of the UHRs to the left and right of the maximum 
of $\omega_{U H}$ are referred to as $x_{l}$ and $x_{r}$, respectively. The trapping region acts as a cavity for the UH plasmons and only permits trapping of certain wave modes, distinguished by phase shifts which are a multiple of $2 \pi$ after each round trip in the trapping region to ensure a single-valued potential. Accounting for the phase shift of $\pi$ associated with the mode conversions at the UHRs during a round trip through the trapping region [42], we arrive at the Bohr-Sommerfeld condition for wave trapping, also found in $[5,6,9,12]$,

$$
\int_{x_{l}}^{x_{r}}\left(k_{1 x}^{+}-k_{1 x}^{-}\right) \mathrm{d} x=\pi(2 m+1)
$$

where $m \in \mathbb{Z}$. For given $\omega_{1}$ and $m$, a mode may be found by varying $k_{1 y}$, which is a conserved quantity due to the homogeneity of the slab along the $y$-direction, such that (4) is satisfied.

As a PDI involves decay of a single pump wave, characterized by subscript 0 , into two daughter waves, characterized by subscripts 1 and 2, energy and momentum conservation in the three-wave process impose the selection rules,

$$
\omega_{0}=\omega_{1}+\omega_{2}, \quad \mathbf{k}_{0}=\mathbf{k}_{1}+\mathbf{k}_{2} .
$$

Taking the pump wave to propagate along the $x$ direction $\left(\mathbf{k}_{0}=k_{0} \mathbf{e}_{x}, k_{0}>0\right.$ for all $\left.x\right)$ and the daughter waves to propagate perpendicular to the $z$ direction, the $\mathbf{k}$ selection rules become

$$
k_{1 x}=k_{0}-k_{2 x}, \quad k_{1 y}=-k_{2 y} .
$$

Since the $k_{x}$ selection rule $\left(k_{1 x}=k_{0}-k_{2 x}\right)$ can only be satisfied at discrete points in the $x$ direction, PDIs generally only lead to finite spatial growth of the daughter wave amplitudes in narrow regions around such points for untrapped daughter waves [1-6]. However, when the pump wave can decay into two trapped daughter waves, the amplification regions are traversed multiple times, allowing such PDIs to cause temporal growth of the daughter wave amplitudes in the trapping region [9].

To keep the analysis relatively simple, we consider decay of the X-mode pump wave to two trapped UH plasmons with identical frequencies, $\omega_{1}=\omega_{2}=\omega_{0} / 2$. This type of PDI may occur when a maximum of $\omega_{U H}$ occurs near the point where $\omega_{0}=2 \omega_{U H}$, i.e., the second-harmonic UHR of the pump wave. We take $k_{1 x}^{ \pm}>0$ and $k_{2 x}^{ \pm}=-k_{1 x}^{ \pm}<0$, which permits the satisfaction of the $k_{x}$ selection rule for the EBW branch of daughter wave 1 and the X-mode branch of daughter wave $2\left(k_{1 x}^{+}=k_{0}-k_{2 x}^{-}\right.$at some points in the trapping region), as seen in pane (a) of figure 1. The trapped modes are found using (4), choosing the $m$ value which minimizes $\left|k_{1 y}\right|=\left|k_{2 y}\right|$. This is done to minimize the convective losses of daughter waves from the pump wave beam along the $y$-direction, since these are determined by the group velocity of the daughter waves along the $y$ direction in the trapping region,

$$
v_{y}=\left|v_{1 y}\right|=\left|v_{2 y}\right|=\left|\frac{\left\langle\partial \mathcal{D}_{1}^{\prime} / \partial k_{y}\right\rangle}{\left\langle\partial \mathcal{D}_{1}^{\prime} / \partial \omega\right\rangle}\right|,
$$

where

$$
\left\langle f_{1}\right\rangle=\frac{1}{N_{1}} \int_{x_{l}}^{x_{r}}\left(\frac{f_{1}^{+}}{\left|\partial \mathcal{D}_{1}^{\prime+} / \partial k_{x}\right|}+\frac{f_{1}^{-}}{\left|\partial \mathcal{D}_{1}^{\prime-} / \partial k_{x}\right|}\right) \mathrm{d} x
$$




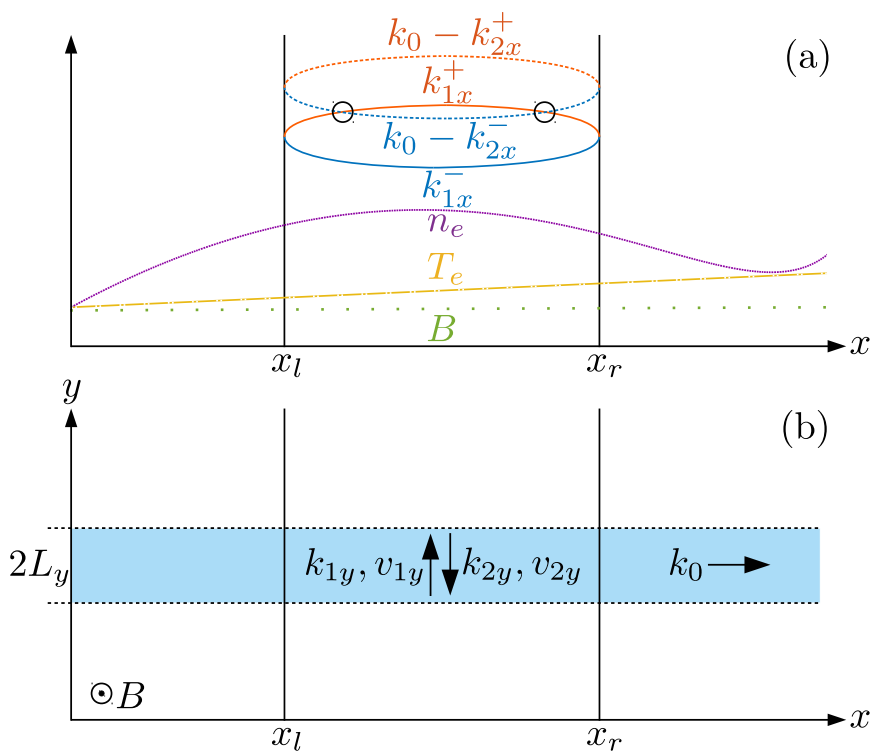

Figure 1. Geometry of the plasma and pump beam used for the theoretical calculations. Pane (a) shows the variation of the plasma parameters, $n_{e}, T_{e}$, and $B$, along the inhomogeneous $x$-direction, along with the dispersion curves of the trapped $\mathrm{UH}$ plasmons; the PDI selection rules are satisfied at points where $k_{1 x}=k_{0}-k_{2 x}$ (marked by black circles). Pane (b) shows the square pump beam as a shaded area, indicates the convective losses of the $\mathrm{UH}$ plasmons along the $y$-direction, and shows $\mathbf{B}$ pointing along the $z$-direction.

$$
N_{1}=\int_{x_{l}}^{x_{r}}\left(\frac{1}{\left|\partial \mathcal{D}_{1}^{\prime+} / \partial k_{x}\right|}+\frac{1}{\left|\partial \mathcal{D}_{1}^{\prime-} / \partial k_{x}\right|}\right) \mathrm{d} x
$$

denotes the average of a function $f$ evaluated at the parameters of daughter wave 1 and weighted by the square amplitude of daughter wave 1, which is proportional to $1 /\left|\partial \mathcal{D}_{1}^{\prime} / \partial k_{x}\right|$ [6], in the trapping region; note that $v_{1 y}=-v_{2 y}$, since $k_{1 y}=-k_{2 y}$ from (6) and $\mathcal{D}$ is an even function of $k_{y}$.

Taking the pump beam to be a uniform square beam of infinite extent along the $x$-direction and width $2 L_{y}$ along the $y$-direction, as shown in pane (b) of figure 1, and neglecting diffraction losses along the $z$-direction, the inital growth rate of the PDI near the second-harmonic UHR, $\gamma$, may be evaluated using (4.85) of [6], which is similar to (6) of [43,

$$
2 Q_{y} L_{y} \cot \left(2 Q_{y} L_{y}\right)=-\frac{2(\gamma+\Gamma) L_{y}}{v_{y}}
$$

$\Gamma=\left\langle\mathcal{D}_{1}^{\prime \prime}\right\rangle /\left\langle\partial \mathcal{D}_{1}^{\prime} / \partial \omega\right\rangle$ is the damping rate of the $\mathrm{UH}$ plasmons in the trapping region and

$$
Q_{y}=\frac{\sqrt{\gamma_{12}(0,0) \gamma_{21}^{*}(0,0)-(\gamma+\Gamma)^{2}}}{v_{y}},
$$

where

$$
\gamma_{12}(0,0) \gamma_{21}^{*}(0,0)=\frac{\omega_{1}^{2}}{4 N_{1}^{2}\left\langle\partial \mathcal{D}_{1}^{\prime} / \partial \omega\right\rangle^{2}} \mid \int_{x_{l}}^{x_{r}} \frac{E_{0 y}(x, 0,0)}{B} \frac{\omega_{p e}^{2} \omega_{c e}^{2} k_{0} k_{1 x}^{+} k_{1 x}^{-}}{\left(4 \omega_{1}^{2}-\omega_{c e}^{2}\right)\left(\omega_{1}^{2}-\omega_{c e}^{2}\right)^{2}}
$$




$$
\times\left.\frac{3+\left(1-\omega_{c e}^{2} / \omega_{1}^{2}\right) / 2}{\sqrt{\partial \mathcal{D}_{1}^{\prime+} / \partial k_{x}} \sqrt{\partial \mathcal{D}_{1}^{\prime-} / \partial k_{x}}} \mathrm{e}^{\mathrm{i} \int_{x_{l}}^{x}\left(k_{0}+k_{1 x}^{-}-k_{1 x}^{+}\right) \mathrm{d} x^{\prime}} \mathrm{d} x\right|^{2}
$$

is the nonlinear coupling factor responsible for the PDI and $E_{0 y}(x, 0,0)$ is the central electric field amplitude of the pump beam along the $y$ direction. The quantity $\gamma_{12}(0,0) \gamma_{21}^{*}(0,0)$ is proportional to the square pump electric field amplitude, meaning that it is also proportional to the pump beam power, $P_{0}$, which may be connected to $E_{0 y}(x, 0,0)$ using the method described in [44. It is therefore possible to write $\gamma_{12}(0,0) \gamma_{21}^{*}(0,0)=\gamma_{12}^{\text {ref }}(0,0) \gamma_{21}^{\text {ref } *}(0,0) P_{0} / P_{0}^{\text {ref }}$, where $\gamma_{12}^{\text {ref }}(0,0) \gamma_{21}^{\text {ref* }}(0,0)$ is the coupling factor evaluated at an arbitrary reference pump beam power, $P_{0}^{\text {ref }}$. We are interested in finding the pump beam power PDI threshold, $P_{0}^{\text {th }}$, which is the point where $\gamma=0$ and $Q_{y}=Q_{y}^{\text {th }}=\sqrt{\gamma_{12}^{\text {ref }}(0,0) \gamma_{21}^{\text {ref } *}(0,0) P_{0}^{\text {th }} / P_{0}^{\text {ref }}-\Gamma^{2}} / v_{y}$, recasting (9) as

$$
2 Q_{y}^{\text {th }} L_{y} \cot \left(2 Q_{y}^{\text {th }} L_{y}\right)=-\frac{2 \Gamma L_{y}}{v_{y}} .
$$

12 is a transcendental equation for $P_{0}^{\text {th }}$, which can be solved numerically once the averages over the trapping region have been performed. It is, however, instructive to solve 12 in an approximate analytical fashion to obtain the dependence of $P_{0}^{\text {th }}$ on the various parameters. To do this, we assume that $\Gamma L_{y} / v_{y} \ll 1$, i.e., that the damping losses of the UH waves in the trapping region are small compared with the convective losses from the pump beam. This requires the left hand side to be numerically small, allowing us to replace $\cot \left(2 Q_{y}^{\text {th }} L_{y}\right)$ by a first order Taylor series around its zeros, $\cot \left(2 Q_{y}^{\text {th }} L_{y}\right) \approx-\left[2 Q_{y}^{\text {th }} L_{y}-\pi(s+1 / 2)\right]$, where $s \in \mathbb{N}_{0}$. Inserting the Taylor series in (12), and rearranging, yields

$$
\left(Q_{y}^{\mathrm{th}} L_{y}\right)^{2}-\frac{\pi}{2}\left(s+\frac{1}{2}\right) Q_{y}^{\mathrm{th}} L_{y}-\frac{\Gamma L_{y}}{2 v_{y}}=0
$$

which is a quadratic equation for $Q_{y}^{\text {th }} L_{y}$ that may be solved, subject to the requirement $Q_{y}^{\text {th }} L_{y}>0$, to give

$$
Q_{y}^{\mathrm{th}} L_{y}=\frac{\pi}{4}\left(s+\frac{1}{2}\right)+\sqrt{\frac{\pi^{2}}{16}\left(s+\frac{1}{2}\right)^{2}+\frac{\Gamma L_{y}}{2 v_{y}}} .
$$

Clearly, $Q_{y}^{\text {th }}$, and hence $P_{0}^{\text {th }}$, is minimized for $s=0$, i.e., at the first zero of the cotangent. Thus, the primary $P_{0}^{\text {th }}$ is obtained by inserting $Q_{y}^{\text {th }}=$ $\sqrt{\gamma_{12}^{\text {ref }}(0,0) \gamma_{21}^{\text {ref } *}(0,0) P_{0}^{\text {th }} / P_{0}^{\text {ref }}-\Gamma^{2}} / v_{y}$ in 14 with $s=0$,

$$
P_{0}^{\text {th }}=\left[\left(\frac{\pi}{8}+\sqrt{\frac{\pi^{2}}{64}+\frac{\Gamma L_{y}}{2 v_{y}}}\right)^{2}+\frac{\Gamma^{2} L_{y}^{2}}{v_{y}^{2}}\right] \frac{v_{y}^{2}}{\gamma_{12}^{\text {ref }}(0,0) \gamma_{21}^{\text {ref } *}(0,0) L_{y}^{2}} P_{0}^{\text {ref }} .
$$

We note that the $P_{0}^{\text {th }}$ given by 15 is equivalent to (4.88) of [6] for $\Gamma=0$, but that the inclusion of the right hand side of (9), which leads to the $\Gamma L_{y} /\left(2 v_{y}\right)$ term in the square root of (15), made here is necessary to realistically evaluate the effect of UH wave damping on $P_{0}^{\text {th }}$. On the other hand, the $P_{0}^{\text {th }}$ of 15 is significantly different from that obtained using (34) of [5] for the reasons discussed in [6]. In section 4 , the $P_{0}^{\text {th }}$ obtained from 12 and 15 are shown to be in good agreement with the experimental $P_{0}^{\text {th }}$. 


\section{Experimental Setup}

The experiments discussed in this paper were carried out at the medium-sized tokamak ASDEX Upgrade, which has a major radius of $1.65 \mathrm{~m}$, a minor radius of $0.5 \mathrm{~m}$, a typical plasma current $I_{p} \sim 1 \mathrm{MA}$, and a typical on-axis toroidal magnetic field $B_{t} \sim-2.5 \mathrm{~T}$ 45]. Specifically, the observations were made using the CTS system [46], which is a heterodyne radiometer utilizing two receivers (referred to as Receiver A and Receiver B [47]), each consisting of a filter bank system sampling at $100 \mathrm{kSamples} / \mathrm{s}$ [48] and a fast-acquisition system sampling a single channel at 6.25 GSamples/s [47], connected to the ECRH waveguide and steerable mirror systems [7]. Rather than the CTS system operating near $105 \mathrm{GHz}$ described in 47,48 , the measurements are made by an upgraded version of the system operating near $140 \mathrm{GHz}$ [20, 49], which is the ECRH frequency used in standard second-harmonic X-mode ECRH at ASDEX Upgrade [7,8]. In addition to the channels sampling the signal near $140 \mathrm{GHz}$, the upgraded system has channels operating near $70 \mathrm{GHz}$ to detect signatures of the waves at around half the ECRH frequency expected in connection with PDIs at the second-harmonic UHR [13]. Figure 2 shows a block diagram of the front-end of the $140 / 70 \mathrm{GHz}$ CTS system at ASDEX Upgrade. The microwave horn of the $140 / 70 \mathrm{GHz}$ CTS system is shared by the $105 \mathrm{GHz}$ CTS system of Receiver A, with a waveguide switch enabling the 140/70 GHz or the 105 $\mathrm{GHz}$ system to be selected [20]. The $140 \mathrm{GHz}$ line contains two notch filters damping stray radiation from the ECRH gyrotrons around $140 \mathrm{GHz}$, a band pass filter to select the frequency range to which the radiometer is sensitive, a PIN variable attenuator enabling operation at various levels of signal strength, an isolator, and a mixing stage of the filtered signal with a $130.5 \mathrm{GHz}$ local oscillator to produce an intermediate frequency (IF) signal that can be digitized; after mixing, the IF signal is amplified and processed using the infrastructure of Receiver A, described in [47]. The $70 \mathrm{GHz}$ line is split off from the $140 \mathrm{GHz}$ line using a directional coupler before the filters. It contains an isolator, along with two low pass filters removing signals above $80 \mathrm{GHz}$, and since the waveguide has a cutoff for frequencies below $59 \mathrm{GHz}$, this selects the frequency range to which the line is sensitive. After the filters, the signal is mixed with that of a 60.01 $\mathrm{GHz}$ local oscillator, producing an IF signal which is amplified and sampled using the infrastructure of Receiver B, presented in [47]; only the mid-IF band (8.16-10.64 GHz) is digitized by the filter bank of Receiver $\mathrm{B}$.

This paper focuses on four ASDEX Upgrade discharges, labeled Discharges 1-4 for easy reference, whose basic parameters and ASDEX Upgrade shot numbers are listed in table 1; plasma scenarios at selected time points from the discharges, calculated using the CLISTE code [50] and integrated data analysis [51], are seen in figure 3 . All discharges have $B_{t}=-2.5 \mathrm{~T}$, leading to a central second-harmonic ECR for the $140 \mathrm{GHz}$ X-mode ECRH radiation (seen in figure 3), sufficient heating power to enter H-mode, and sufficiently high $n_{e}$ for the second-harmonic UHR to occur at the plasma edge, which is a necessary condition for the PDIs discussed above to occur in connection with relatively small edge density perturbations; additionally, the ELMs found in the 


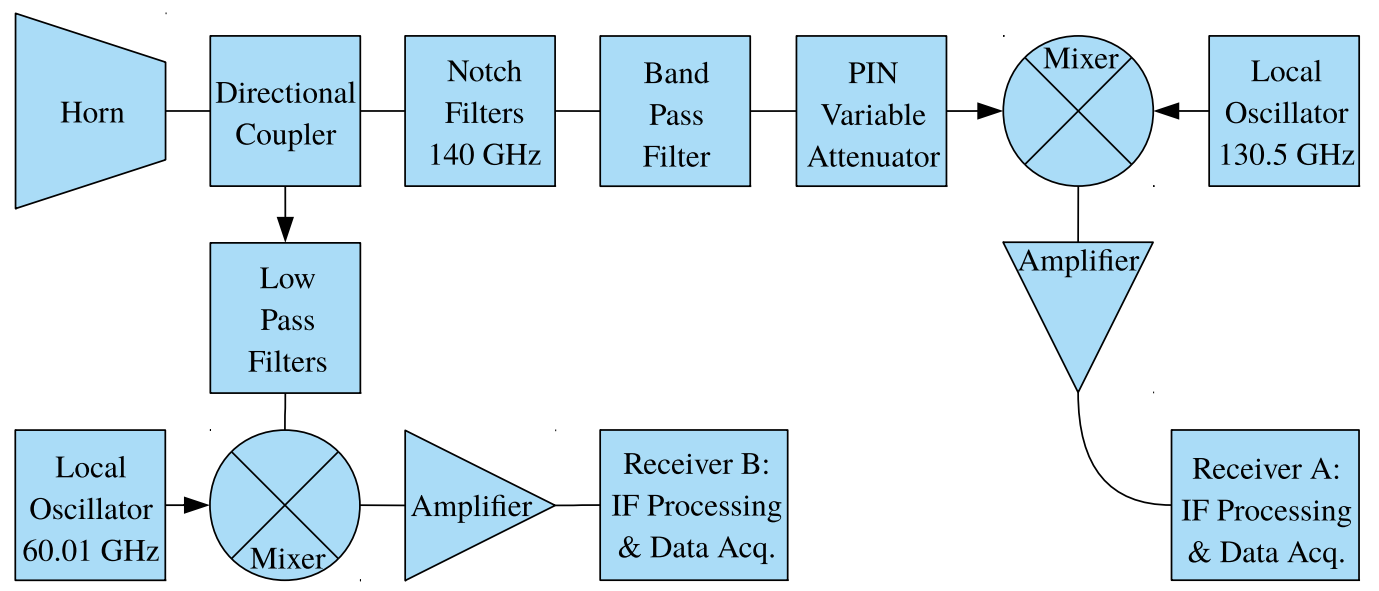

Figure 2. Block diagram of the $140 / 70 \mathrm{GHz}$ CTS system front-end at ASDEX Upgrade; the arrows represent isolators. The waveguides have a cutoff for frequencies below $59 \mathrm{GHz}$, the low pass filters block frequencies above $80 \mathrm{GHz}$, the notch filters block frequencies in the range $139.7-140.1 \mathrm{GHz}$, and the band pass filter transmits frequencies in the range $135-145 \mathrm{GHz}$. The signals in the frequency ranges $68.1-70.7$ $\mathrm{GHz}$ and $135-145 \mathrm{GHz}$ are sampled by filter bank systems at $100 \mathrm{kSamples} / \mathrm{s}$; the signals in the frequency ranges $68.16-70.50 \mathrm{GHz}$ and $138.4-141.2 \mathrm{GHz}$ are additionally sampled by fast-acquisition systems at 6.25 GSamples/s.

\begin{tabular}{|r|c|c|c|c|}
\hline Discharge \# & 1 & 2 & 3 & 4 \\
\hline ASDEX Upgrade Shot \# & 37156 & 37155 & 32027 & 33616 \\
\hline$I_{p}[\mathrm{MA}]$ & 0.8 & 0.8 & 1.0 & 0.8 \\
\hline$B_{t}[\mathrm{~T}]$ & -2.5 & -2.5 & -2.5 & -2.5 \\
\hline Central Chord $\bar{n}_{e}\left[10^{19} \mathrm{~m}^{-3}\right]$ & $5.8-7.6$ & $5.9-7.5$ & 8.0 & 6.1 \\
\hline Neutral Beam Power $[\mathrm{MW}]$ & 2.5 & 2.5 & 5.0 & 2.5 \\
\hline ECRH Power $[\mathrm{MW}]$ & 2.0 & $1.5-1.8$ & 0.6 & 1.7 \\
\hline ICRH Power $[\mathrm{MW}]$ & 0 & 0 & $0-1.8$ & 0 \\
\hline
\end{tabular}

Table 1. Basic parameters during the relevant H-mode phases of the ASDEX Upgrade discharges considered in this Paper.

discharges are all of type I [52].

Discharges 1 and 2 are similar in terms of plasma shape, $I_{p}$, and heating. Further, Discharges 1 and 2 both include an $n_{e}$ scan during the time interval $3.5-7.5 \mathrm{~s}$, indicated by the range of the line-averaged $n_{e}, \bar{n}_{e}$, from the central chord (H-1) of the ASDEX Upgrade interferometer system [53] in table 1, to allow the impact of changing $n_{e}$ on the PDI characteristics to be investigated; from figure 3 it is evident that the second-harmonic UHR remains close to plasma edge throughout the $n_{e}$ scan, as the plasma scenario of Discharge 1 shows the situation near the end of the $n_{e}$ scan $\left(\bar{n}_{e}=7.2 \times 10^{19} \mathrm{~m}^{-3}\right)$, while the plasma scenario of Discharge 2 shows the situation before the $n_{e}$ scan $\left(\bar{n}_{e}=6.1 \times 10^{19} \mathrm{~m}^{-3}\right)$. The main difference between Discharges 1 and 2 is that the probe gyrotron, whose beam overlaps with the CTS receiver view near the 


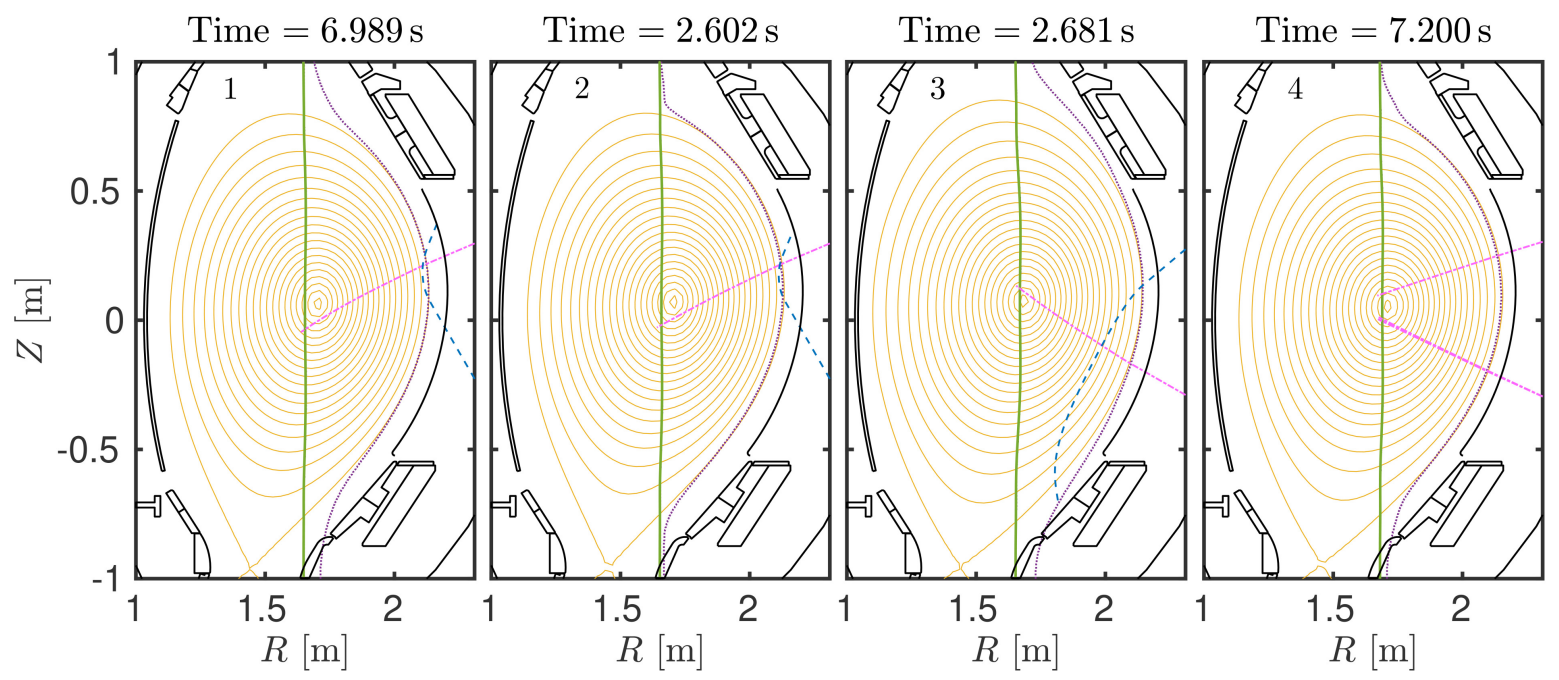

Figure 3. Plasma scenarios of Discharges $1-4$ (left to right) at selected time points. Here, $(R, Z)$ are cylindrical coordinates, green thick lines are the $140 \mathrm{GHz}$ secondharmonic ECR, regular black lines are the plasma vessel components, thin golden lines are the flux surfaces of the core plasma, dashed-dotted pink lines are probe gyrotron beams, dashed blue lines are CTS receiver views, and purple fine dotted lines are the $140 \mathrm{GHz}$ second-harmonic warm UHR.

plasma edge, is run in continuous wave mode in Discharge 1, while it is subject to fast analog modulations of $P_{0}$, indicated by the ECRH power range in table 1 and resembling those from [54], in Discharge 2 to allow determination of $P_{0}^{\text {th }}$. In Discharges 1 and 2, the polarizer of the CTS receiver [55] is set such that the $70 \mathrm{GHz}$ and $140 \mathrm{GHz}$ systems both accept roughly $50 \% \mathrm{X}$-mode polarized and $50 \% \mathrm{O}$-mode polarized radiation. This allows all strong microwave signals to be identified at the cost of detailed information about the polarization of the strong signals; we do, however, note that the signals near $70 \mathrm{GHz}$ are observed for polarizer settings corresponding to pure X-mode as well as pure O-mode, based on other discharges, indicating the occurrence of processes leading to cross-polarization.

Discharge 3 has a larger $I_{p}, \bar{n}_{e}$, and more neutral beam power than Discharges 1 and 2. Additionally, ion cyclotron resonance heating (ICRH) is introduced in the latter part of the discharge, as indicated by the ICRH power range in table 1, which changes the poloidal velocity of the ELM filaments [56 58]. Further, Discharge 3 includes a scan of the receiver view, allowing the location of the microwave source to be pin-pointed. Since Discharge 3 is an older discharge, it only includes the $140 \mathrm{GHz}$ CTS system and has the receiver located above the midplane, as opposed the receiver location below the midplane in Discharges 1 and 2; the polarizer is set such that the $140 \mathrm{GHz}$ system accepts predominantly $\mathrm{X}$-mode radiation.

Finally, Discharge 4 is the discharge for which an ELM crash is simulated using the nonlinear MHD code JOREK [34 36]. It matches Discharges 1 and 2 before the start of the $n_{e}$ scans in terms of plasma shape, $I_{p}, \bar{n}_{e}$, and heating, but does not include measurements by the CTS system, so figure 3 shows the beams of all ECRH gyrotrons. 


\section{Experimental and Numerical Results}

Now that the theory of PDIs near the second-harmonic UHR and the experimental setup used to detect them at ASDEX Upgrade has been discussed, we proceed to present the experimental results. This is done by covering the most important aspects of the discharges from table 1 and figure 3 in individual subsections.

\section{Discharge 1: General Characteristics}

Since the probe gyrotron is operated in continuous wave mode in Discharge 1, this discharge is ideal for presenting the general microwave features observed near the ECRH frequency $(140 \mathrm{GHz})$ and half the ECRH frequency $(70 \mathrm{GHz})$. Microwave bursts are clearly visible in the spectral power density (SPD) of the filter bank channels at 69.61 $\mathrm{GHz}$ and $139.55 \mathrm{GHz}$, shown in figure 4, after the probe gyrotron is switched on at $1.5 \mathrm{~s}$. Just after the probe gyrotron is switched on, quasi-continuous strong signals are observed at both $69.61 \mathrm{GHz}$ and $139.55 \mathrm{GHz}$. These signals occur while the plasma is still in L-mode, meaning that they are most likely related to the blobs which are continually ejected from the plasma in connection with L-mode turbulence [24]; in the discussion of Discharge 2, these signals are shown to have power a threshold, which justifies identifying their origin with PDIs. As the plasma enters H-mode, the strength of the quasi-continuous signals is greatly reduced, consistent with the suppression of edge turbulence in $\mathrm{H}$-mode relative to L-mode [59]. In place of the quasi-continuous strong signals are quasi-periodic microwave bursts coinciding with the occurrence of ELMs, as indicated by the spikes of the divertor current in figure 4. These results are in agreement with previous observations of microwave bursts from the edge of ASDEX Upgrade plasmas near $140 \mathrm{GHz}[6,20,21]$. We note that the values of the SPD near 70 $\mathrm{GHz}$ and $140 \mathrm{GHz}$ observed in connection with the bursts are both $\sim 100 \mathrm{keV}$.

In order to give an overview of the $n_{e}$ scan and its impact on the microwave signal near half the ECRH frequency, we show the development of $\bar{n}_{e}$ from two chords of the ASDEX Upgrade interferometer system [53], one which passes close to the plasma center (H-1) and one which passes through the plasma edge (H-5), in pane (a) of figure 5. along with the SPD from the $70 \mathrm{GHz}$ filter bank CTS system in pane (b) of figure 5 pane $\left(\mathrm{b}^{*}\right)$ of figure 5 shows the mean SPD for the H-mode plasma before the start of the $n_{e}$ scan $(2-3 \mathrm{~s})$ and during the latter part of the $n_{e}$ scan $(5-7 \mathrm{~s})$ for easier identification of the various peaks. We note that strong signals only occur while the probe gyrotron is on $(1.5-7.5 \mathrm{~s})$, as well as the existence of strong signals in particular frequency bands, which are modified by the $n_{e}$ scan from $3.5 \mathrm{~s}$ onward. The narrow bands containing strong signals in figure 5 are related to inter-ELM modes, which can lead to a local $n_{e}$ maximum allowing the $\mathrm{UH}$ wave trapping necessary for the PDIs to occur near the pedestal top [23], and are partially replaced by broadband features during ELMs, to be discussed in connection with figure 6. Two bands (1 and $\left.1^{*}\right)$ are visible just above and just below $70 \mathrm{GHz}$; they have SPD maxima at $70.09 \mathrm{GHz}$ and 69.77 GHz before the $n_{e}$ scan and at $70.17 \mathrm{GHz}$ and $69.69 \mathrm{GHz}$ during the latter part of 


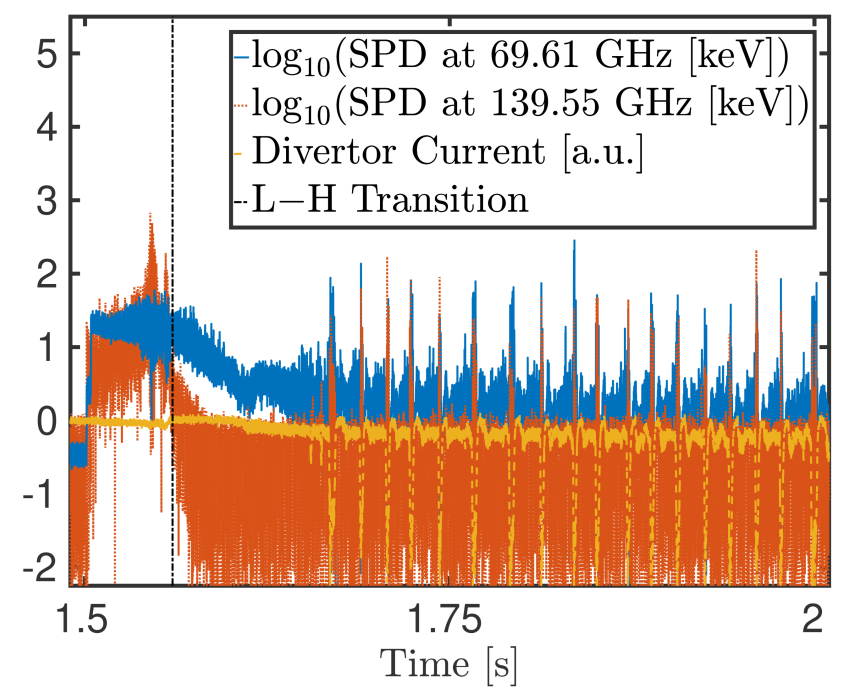

Figure 4. SPD at $69.61 \mathrm{GHz}$ (solid line) and $139.55 \mathrm{GHz}$ (fine dotted line) from the filter bank CTS systems $\left(1 \mathrm{keV}=1.602 \times 10^{-16} \mathrm{~J}\right)$, along with the divertor current (dashed line), in Discharge 1; the $\mathrm{L}-\mathrm{H}$ transition is marked by the dashed-dotted line. The correlation of microwave bursts with ELMs is clearly visible.

the $n_{e}$ scan, as seen in pane $\left(\mathrm{b}^{*}\right)$ of figure 5. Adding the frequencies of the SPD maxima of bands 1 and $1^{*}$, while accounting for the channel widths, yields $139.86 \pm 0.08 \mathrm{GHz}$, which agrees with the probe gyrotron frequency of $139.94 \mathrm{GHz}$, seen in figure 6. It is thus possible to interpret bands 1 and $1^{*}$ as signatures of the primary daughter waves, and this interpretation is further justified by the increasing frequency shift between the two bands with increasing $n_{e}$, in agreement with theoretical expectations [22]. The two lower bands (2 and 3) in figure 5 are down-shifted by $880 \mathrm{MHz}$ and $2 \times 880 \mathrm{MHz}$ from band 1 throughout the discharge, while the upper band appearing during the $n_{e}$ scan $\left(2^{*}\right)$ is shifted by $880 \mathrm{MHz}$ from band $1^{*}$. We can therefore interpret these bands as beat waves created by mixing of one of the daughter waves with an $880 \mathrm{MHz}$ wave, e.g., an ion Bernstein wave, excited in a secondary PDI, or as the direct products of such a secondary PDI [10]. The precise nature of the signals reaching the CTS receiver is discussed in section 5 .

Returning to the signals observed in connection with ELMs, we consider the signals measured by the fast-acquisition CTS systems during an ELM before the $n_{e}$ scan (from $2.059-2062 \mathrm{~s}$ ) and an ELM near the end of the $n_{e}$ scan (from 6.987-6.990 s) in figure 6. The bands of figure 5 are visible before the start of the ELM (indicated by the drop of the divertor current) in the data from the $70 \mathrm{GHz}$ fast-acquisition system, shown in panes (1a) and (1b) of figure6. During the ELM, the bands, particularly the ones near $70 \mathrm{GHz}$, are weakened and augmented by strong spikes in the frequency regions between the bands. The signals near $140 \mathrm{GHz}$, seen in panes (2a) and (2b) of figure 6, are in agreement with previous observations [6]. Before the $n_{e}$ scan, a line down-shifted by 1.5 $\mathrm{GHz}$ from the ECRH frequency is visible during the inter-ELM and early ELM phases, which may be attributed to combination of the wave slightly above $70 \mathrm{GHz}$ (band 1 of 


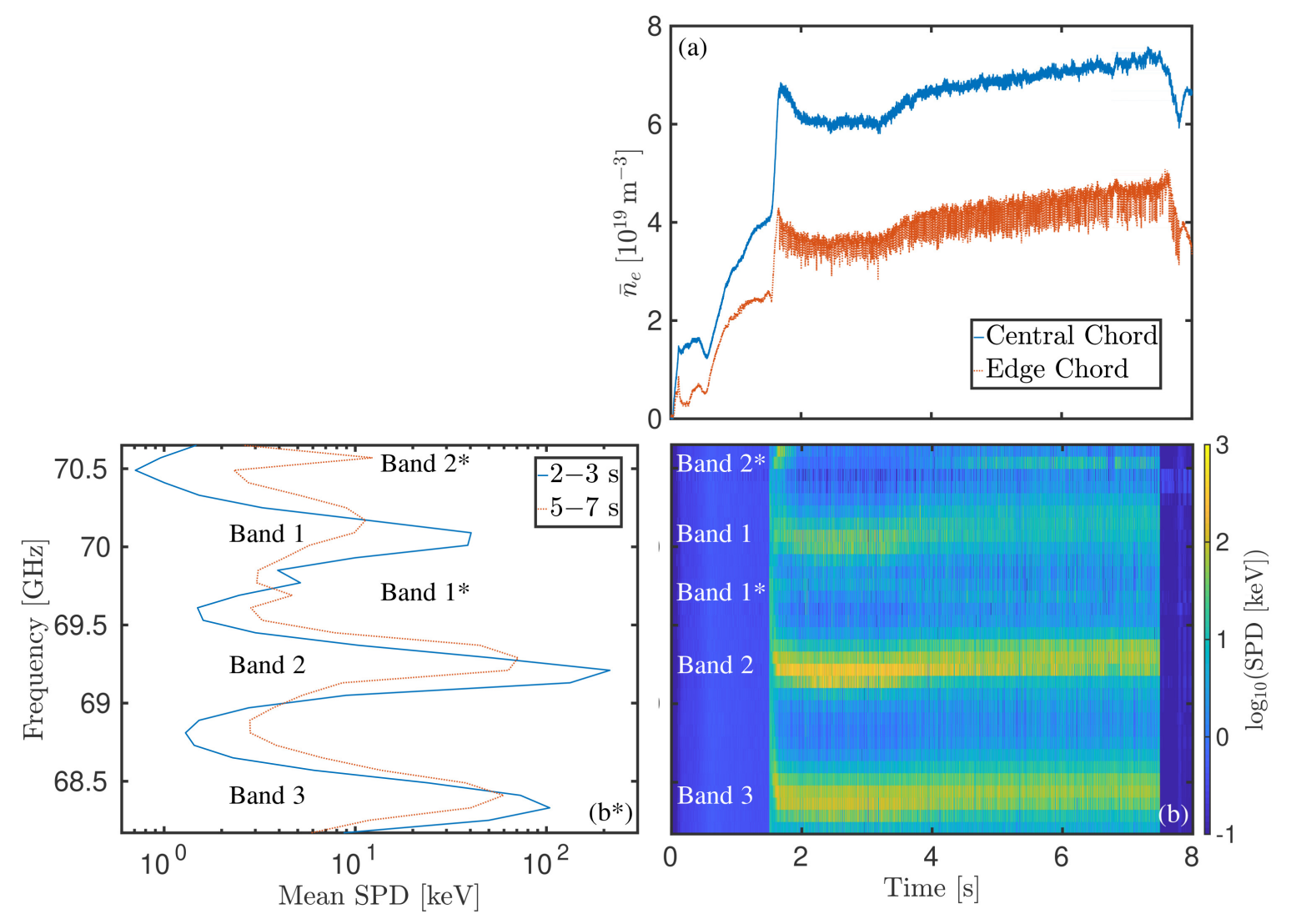

Figure 5. Pane (a) shows $\bar{n}_{e}$ measured by an interferometer chord passing through the plasma center and edge versus time, pane (b) shows the SPD measured by the filter bank of the $70 \mathrm{GHz}$ CTS system versus time, and pane (b*) shows the mean SPD measured by the filter bank of the $70 \mathrm{GHz}$ CTS system from 2-3 s and 5-7 s in Discharge 1 . The frequency bands with strong signals are marked $\left(1,1^{*}, 2,2^{*}, 3\right)$ for easy reference.

figure 5) with the wave near $68.4 \mathrm{GHz}$ (band 3 of figure 5) [10]; this interpretation is also consistent with fact that the signals in the $140 \mathrm{GHz}$ CTS system occur simultaneously with increases of the $68.4 \mathrm{GHz}$ line strength. During the latter part of the $n_{e}$ scan, the inter-ELM signal is virtually non-existent in the $140 \mathrm{GHz}$ CTS system and the downshifted line only occurs briefly during the early ELM phase, in agreement with previous observations [6], as well as the general reduction of the strength of the lines in the 70 GHz CTS system visible in figure 5 and panes (1a) and (1b) of figure 6. Both before and throughout the $n_{e}$ scan, broadband spikes occur during the ELM phase. The spikes in the $140 \mathrm{GHz}$ CTS system appear to be correlated with the spikes in the $70 \mathrm{GHz}$ CTS system, although they are less frequent since they originate from a higher-order PDI than the spikes in the $70 \mathrm{GHz}$ CTS system [10], as demonstrated below. We again note that SPD values $\sim 100 \mathrm{keV}$ are observed for the spikes in both frequency ranges.

To investigate the nature of the spikes in the $70 \mathrm{GHz}$ and $140 \mathrm{GHz}$ CTS systems during ELMs, we compute the spectral power (SP), by integrating the SPD from the fast-acquisition systems, in the frequency ranges where spikes occur in connection with 

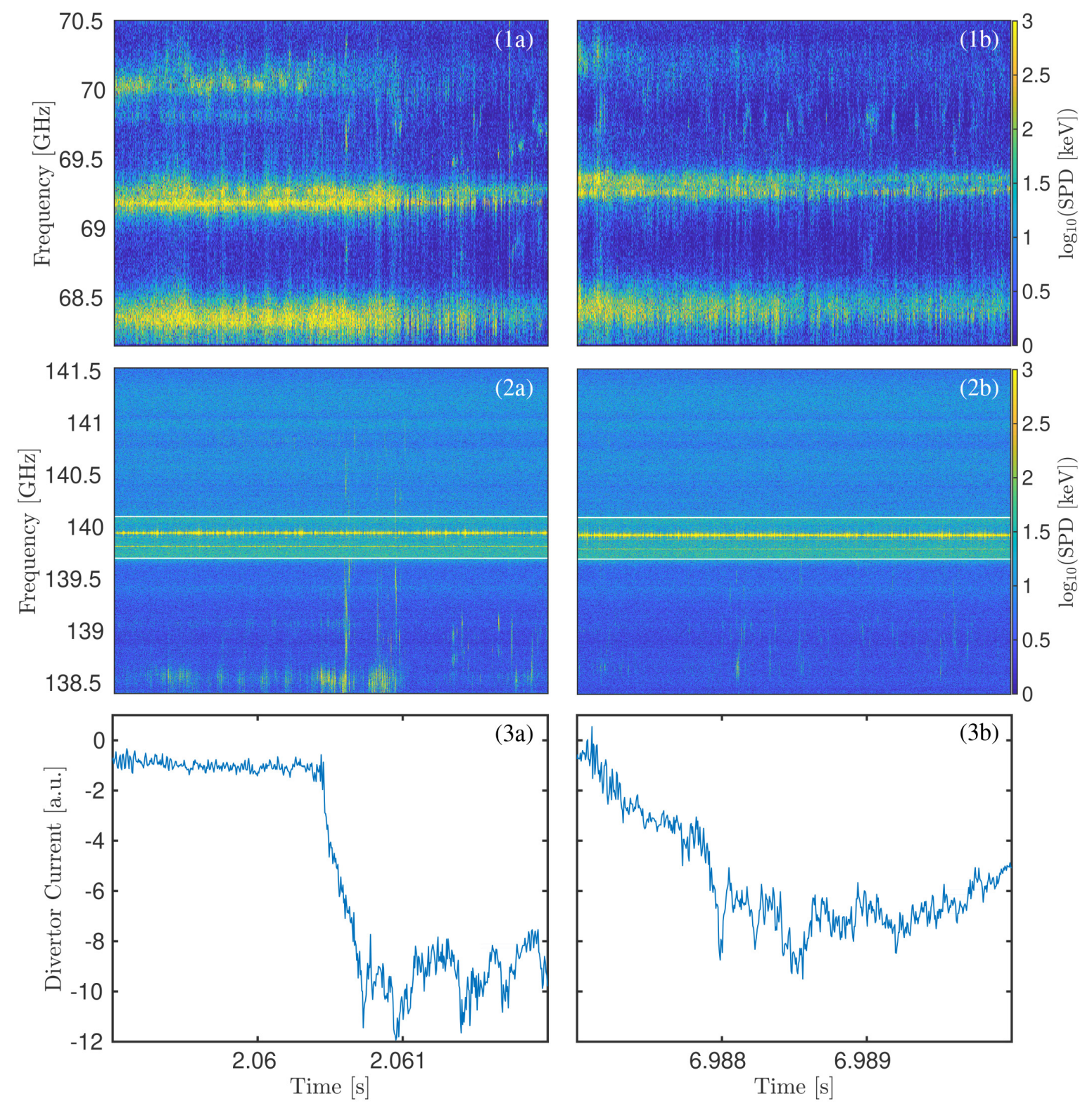

Figure 6. Microwave and ELM signals during an ELM with low $n_{e}$ (at 2.059-2.062 s) and one with high $n_{e}$ (at 6.987-6.990 s) in Discharge 1. Panes (a) are related to the ELM at low $n_{e}$, while panes (b) are related to the ELM at high $n_{e}$. Panes (1a) and (1b) show the fast-acquisition CTS signal near $70 \mathrm{GHz}$. Panes (2a) and (2b) show the same signals near $140 \mathrm{GHz}$; the white lines mark the frequency edges of the notch filter around the ECRH frequencies and the strong signal near $139.94 \mathrm{GHz}$ originates from the probe gyrotron. Panes (3a) and (3b) show the divertor current. 


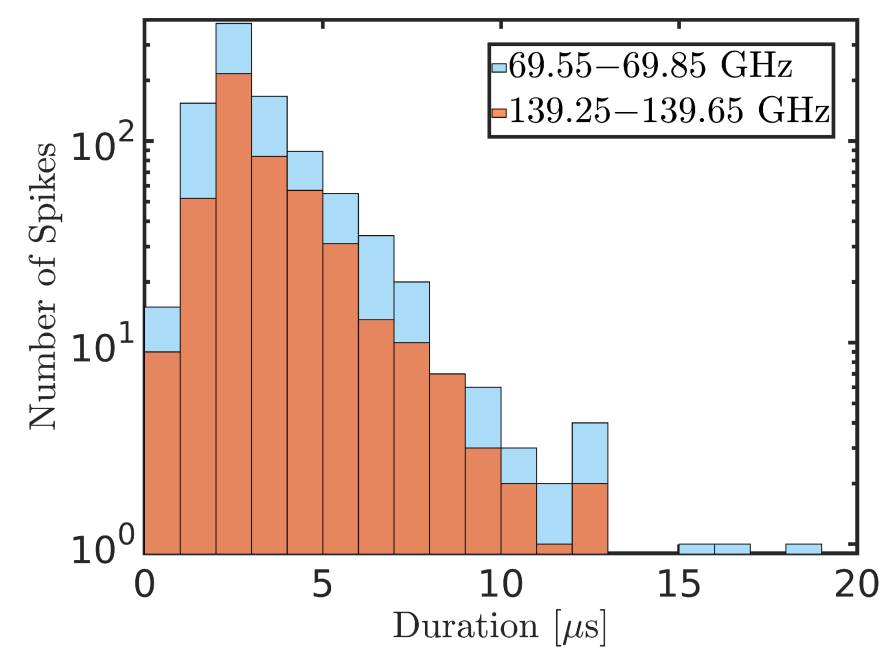

Figure 7. Histograms of the microwave spike duration in the frequency ranges 69.55-69.85 GHz and 139.25-139.65 GHz in Discharge 1.

ELMs (69.55-69.85 GHz for the $70 \mathrm{GHz}$ system and $139.25-139.65 \mathrm{GHz}$ for the 140 $\mathrm{GHz}$ system). The spikes are identified by first smoothing the measured SP using the smoothdata function from MATLAB to reduce the SP noise. We then apply the findpeaks function from MATLAB to the smoothed SP, with the requirement that the prominence of the spikes exceed a set threshold to avoid the inclusion of spurious spikes. Histograms of the duration of the spikes (full-width at half-prominence) in the frequency ranges $69.55-69.85 \mathrm{GHz}$ and $139.25-139.65 \mathrm{GHz}$ are shown in figure 7. Overall, the spike duration distributions are very similar. This is further substantiated by the similar statistics of the spike duration distributions in the frequency ranges $69.55-69.85 \mathrm{GHz}$ and $139.25-139.65 \mathrm{GHz}$, e.g., the means of $3.31 \pm 0.06 \mu \mathrm{s}$ and $3.33 \pm 0.08 \mu \mathrm{s}$, the standard deviations of $1.92 \pm 0.04 \mu \mathrm{s}$ and $1.78 \pm 0.06 \mu \mathrm{s}$, and the maximum probability density from $2-3 \mu \mathrm{s}$ (in both cases), respectively. Based on figure 7 , it is also clear that there are more spikes from $69.55-69.85 \mathrm{GHz}$ than from $139.25-139.65 \mathrm{GHz}$ in all duration intervals (except the one from $8-9 \mu$ s where both histograms record 7 spikes). The above facts indicate that the spikes have similar origins and that the spikes near $70 \mathrm{GHz}$ appear more readily than the spikes near $140 \mathrm{GHz}$, consistent with them originating from a lower-order PDI. This point may be more conclusively demonstrated by application of the conditional averaging technique, which is well-known in connection with the analysis of turbulent structures, such as blobs and eddies [24,60].

Conditional averaging involves taking the average of a signal around time points where the signal satisfies a particular condition, in the present case the condition by which spikes are identified in figure 7, to construct a typical signal associated with the spikes in the $70 \mathrm{GHz}$ and $140 \mathrm{GHz}$ CTS systems. Defining $\tau_{69.55-69.85 \mathrm{GHz}}$ as the time relative to the spikes identified in the frequency range $69.55-69.85 \mathrm{GHz}$ and $\tau_{139.25-139.65 \mathrm{GHz}}$ as the same quantity for the spikes identified in the frequency range $139.25-139.65 \mathrm{GHz}$, the conditional averages of the SP in the frequency ranges 

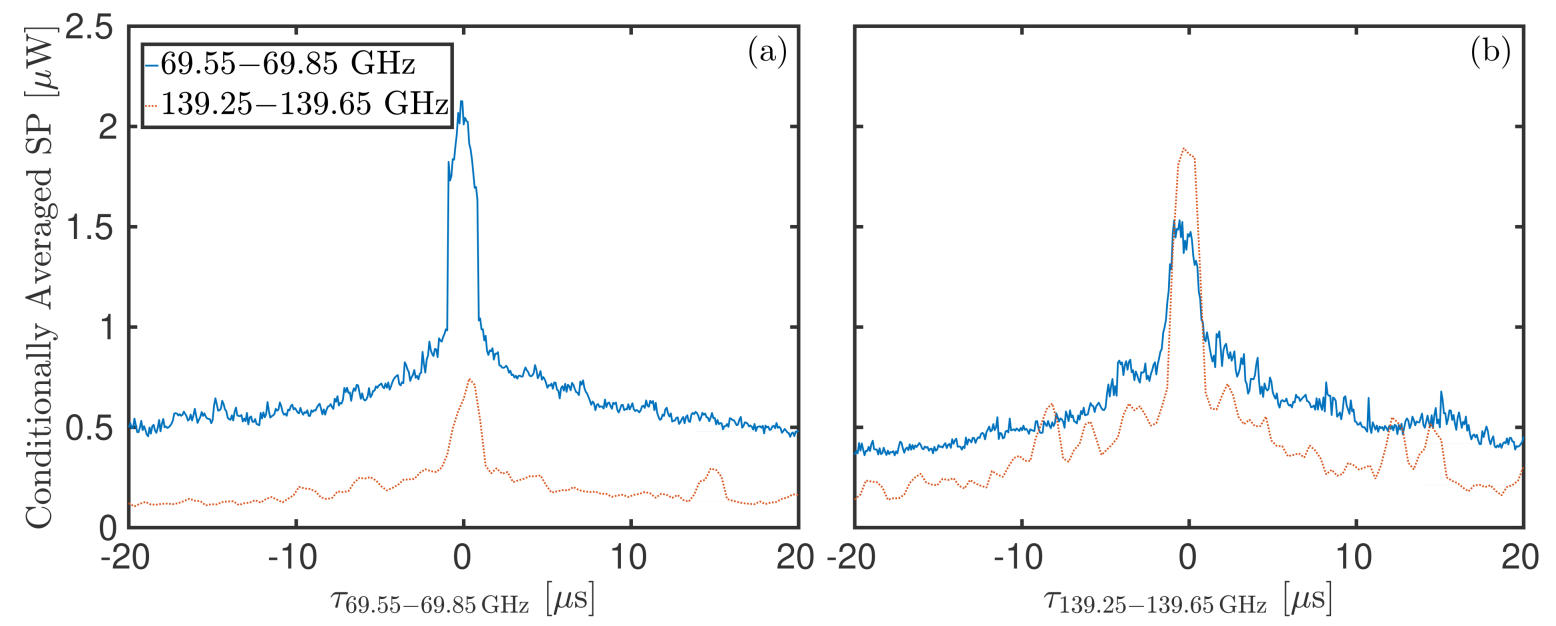

Figure 8. Conditionally averaged SP in the frequency ranges $69.55-69.85 \mathrm{GHz}$ and 139.25-139.65 GHz. Pane (a) shows the conditional averages versus $\tau_{69.55-69.85 \mathrm{GHz}}$, while pane (b) shows the conditional averages versus $\tau_{139.25-139.65 \mathrm{GHz}}$. In both cases, spikes in one frequency range are related to spikes in the other. Spikes in the frequency range $139.25-139.65 \mathrm{GHz}$ appear to predict spikes in the frequency range $69.55-69.85$ $\mathrm{GHz}$ more reliably than the inverse.

69.55-69.85 $\mathrm{GHz}$ and $139.25-139.65 \mathrm{GHz}$ seen in figure 8 are obtained. Pane (a) of figure 8 shows the expected significant increase of the SP from $69.55-69.85 \mathrm{GHz}$ around $\tau_{69.55-69.85 \mathrm{GHz}}=0$, while the $\mathrm{SP}$ from $139.25-139.65 \mathrm{GHz}$ shows a smaller increase, indicating that spikes in the frequency range $69.55-69.85 \mathrm{GHz}$ are only weakly correlated with spikes in the frequency range $139.25-139.65 \mathrm{GHz}$; this is in agreement with the fact that the frequency range $69.55-69.85 \mathrm{GHz}$ contains approximately twice as many spikes as the frequency range $139.25-139.65 \mathrm{GHz}$ based on figure 7 . By contrast, pane (b) of figure 8 shows a significant increase of the SP in both frequency ranges around $\tau_{139.25-139.65 \mathrm{GHz}}=0$, indicating that spikes in the frequency range $139.25-139.65 \mathrm{GHz}$ have a strong correlation with spikes in the frequency range $69.55-69.85 \mathrm{GHz}$; it is also noted that the SP in the two frequency ranges reach similar values $(\sim 2 \mu \mathrm{W})$ during the spikes, in agreement with the similar values of the SPD observed in figures 4 and 6 . As stated above, this supports the interpretation that the spikes near $70 \mathrm{GHz}$ originate from a lower-order PDI than the spikes near $140 \mathrm{GHz}$, since in this case spikes near 140 $\mathrm{GHz}$ imply the occurrence of spikes near $70 \mathrm{GHz}$, while the inverse is not necessarily true. We further observe that the conditionally averaged spikes all have full-widths at half-prominence of approximately $2 \mu \mathrm{s}$, which coincides with the maximum probability density of the spike widths in figure 7. Finally, as will be shown in the discussion of the JOREK simulation of Discharge 4, the spike duration distributions indicated by figures 7 and 8 are consistent with the passage time of a region allowing trapping of $\mathrm{UH}$ plasmons at half the ECRH frequency through an ECRH beam at ASDEX Upgrade, meaning that PDIs near the second-harmonic UHR can account for the spikes. 


\section{Discharge 2: Half-Frequency Signal and PDI Power Threshold}

With the general features of the signals observed from the plasma edge established, we now turn to the demonstration of an experimental probe gyrotron PDI power threshold, $P_{0}^{\text {th }}$, which will be compared with the theoretical estimates of 12 and $(15)$ for the waves near half the ECRH frequency in the discussion of Discharge 4. This is done using the analog modulations of $P_{0}$ from Discharge 2, which are similar to the ones described in [54], but run from $150-530 \mathrm{~kW}$, rather than $0-310 \mathrm{~kW}$.

Before discussing $P_{0}^{\text {th }}$ in connection with ELMs, we consider the signal measured by the fast-acquisition CTS systems just after the probe gyrotron is switched on in figure 9. As seen in pane (a) of figure 9, a chirping narrow line, labeled line 1, appears around $70 \mathrm{GHz}$. Comparing the chirping of line 1 with that of the probe gyrotron line, labeled line 0 , in pane (b) of figure 9, which is caused by the analog modulation of $P_{0}$, it is clear that line 1 may be interpreted as a signal originating directly from the half-frequency waves excited by the primary PDI in the blobs associated with L-mode turbulence [24]; this is further substantiated by the fact that line 1 is only visible for low probe gyrotron frequencies, corresponding to high $P_{0}$. We discuss the precise nature of line 1 in section 5. Apart from lines 1 and 0 , broadband signals (bands 1 and 0 in figure 9) are present below $70 \mathrm{GHz}$ and $140 \mathrm{GHz}$, respectively, just after the probe gyrotron is switched on. These signals are interpreted as the L-mode blob equivalents of the spikes observed during H-mode ELMs, which are also discussed in section 5. Their quasi-continuous nature during the high $P_{0}$ phases, as opposed to the separate spikes observed during ELMs, reflects the continual ejection of blobs in L-mode. Additionally, the SPD of bands 0 and 1 are comparable $(\sim 10 \mathrm{keV})$. We note that band 1 appears before the signals with a greater down-shift and band 0 , indicating the occurrence of a cascade of PDIs with the signals in band 1 being of a lower order, in agreement with theoretical predictions [10] and the discussion of Discharge 1. Pane (b) of figure 9 also shows that a secondary heating gyrotron is switched on after the probe gyrotron. In spite of this, the signals in bands 1 and 0 are still clearly correlated with the chirping of the probe gyrotron frequency, indicating that the signals primarily originate from the overlap between the probe gyrotron beam and the CTS receiver view, consistent with what is expected for PDI-generated signals; the signals in band 1 appear to be somewhat more affected by the secondary gyrotron than the signals in band 0 .

To demonstrate that the signals just after the probe gyrotron is switched on are due to PDIs, rather than effects with a linear dependence on $P_{0}$, we plot the SP in the frequency ranges of line $1(69.975-70.025 \mathrm{GHz})$, band $1(69.65-69.95 \mathrm{GHz})$, and band $0(139.25-139.65 \mathrm{GHz})$ versus $P_{0}$ in figure 10 . The SPs only show significant deviations from the background level present before the probe gyrotron is switched on above a critical $P_{0}$, which we identify as the experimental $P_{0}^{\text {th }}$, indicating that they can all be attributed to PDIs. In order to have an objective measure, we compute SP thresholds of $20 \mathrm{SP}$ standard deviations above the mean SP before the gyrotron is switched on and define $P_{0}^{\text {th }}$ as the lowest $P_{0}$ values for which the SP thresholds are exceeded; the 

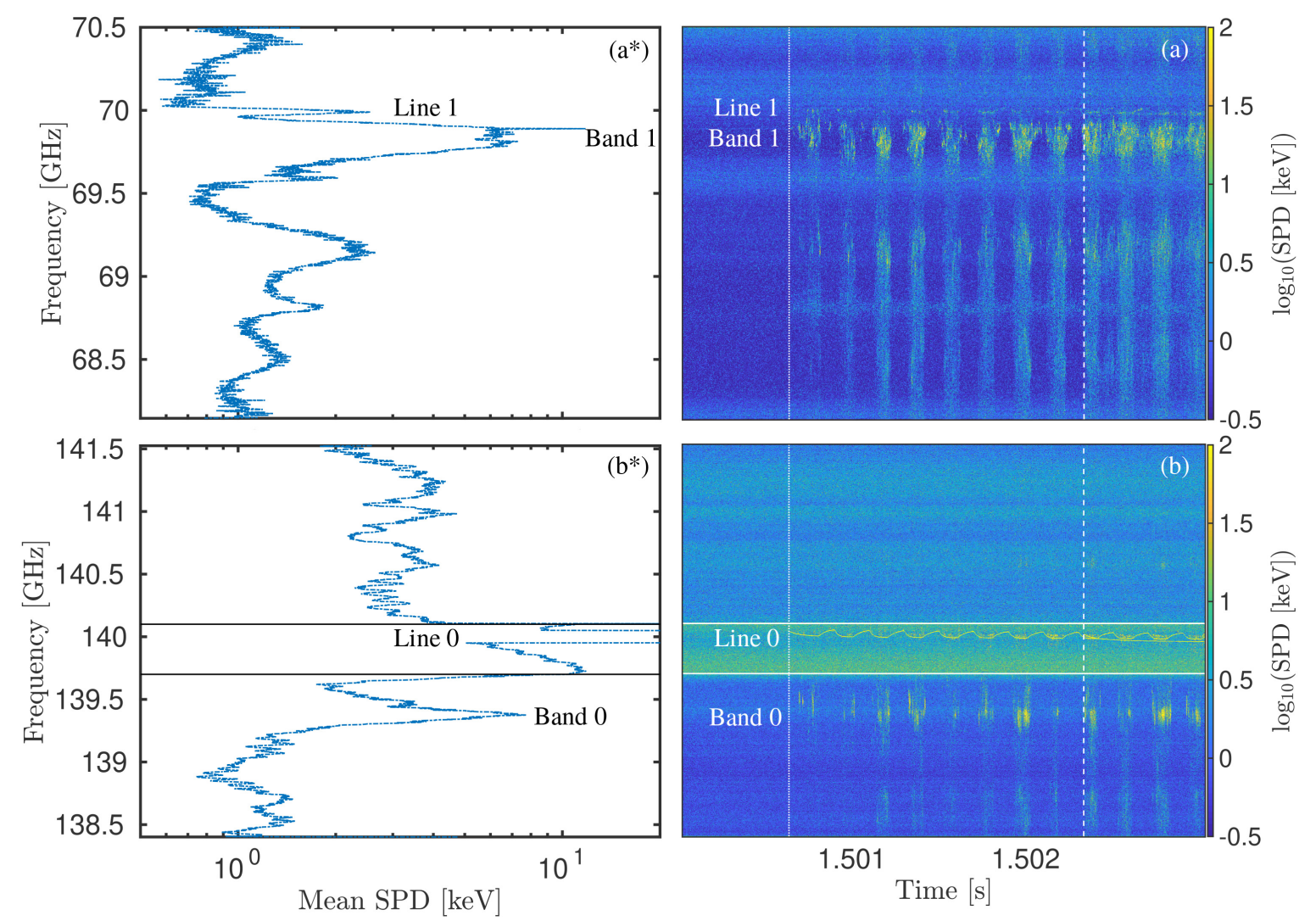

Figure 9. SPD from the fast-acquisition CTS systems just after the probe gyrotron is switched on in Discharge 2. Pane (a) shows the signal near $70 \mathrm{GHz}$, while pane (b) shows the signal near $140 \mathrm{GHz}$; the solid lines mark the frequency edges of the notch filter around the ECRH frequencies, the fine dotted lines mark the time point at which the probe gyrotron is switched on, and the dashed lines mark the time point at which the secondary heating gyrotron is switched on. Panes $\left(a^{*}\right)$ and $\left(b^{*}\right)$ show the mean SPD near $70 \mathrm{GHz}$ and $140 \mathrm{GHz}$, respectively, after the probe gyrotron is switched on. The lines at the ECRH frequency and half the ECRH frequency are marked $(0,1$, respectively), along with the bands down-shifted relative to the ECRH frequency and half the ECRH frequency (also 0, 1, respectively), for easy reference.

SP thresholds and $P_{0}^{\text {th }}$ for line 1 , band 1 , and band 0 are seen in figure 10 . Line 1 and band 1 have similar values of $P_{0}^{\text {th }}, 127 \mathrm{~kW}$ and $103 \mathrm{~kW}$, respectively, which we interpret as estimates of the primary $P_{0}^{\text {th }}$. On the other hand, the signal in band 0 has a higher $P_{0}^{\text {th }}=212 \mathrm{~kW}$, further supporting the interpretation that this signal originates from a higher-order PDI, as expected theoretically [10] and based on the discussion of Discharge 1.

We now determine $P_{0}^{\text {th }}$ of the microwave spikes observed in connection with ELMs, which is directly compared with the theoretical values of $(12)$ and $(15)$ in the discussion of Discharge 4. While it is possible to use a technique similar to that of figure 10 for computing $P_{0}^{\text {th }}$ of the ELM spikes, the highly intermittent nature of the strong signals and the lack of a $P_{0}=0$ reference during the analog modulations, which run from $150-530 \mathrm{~kW}$, favor a different method. We identify the microwave spikes using 

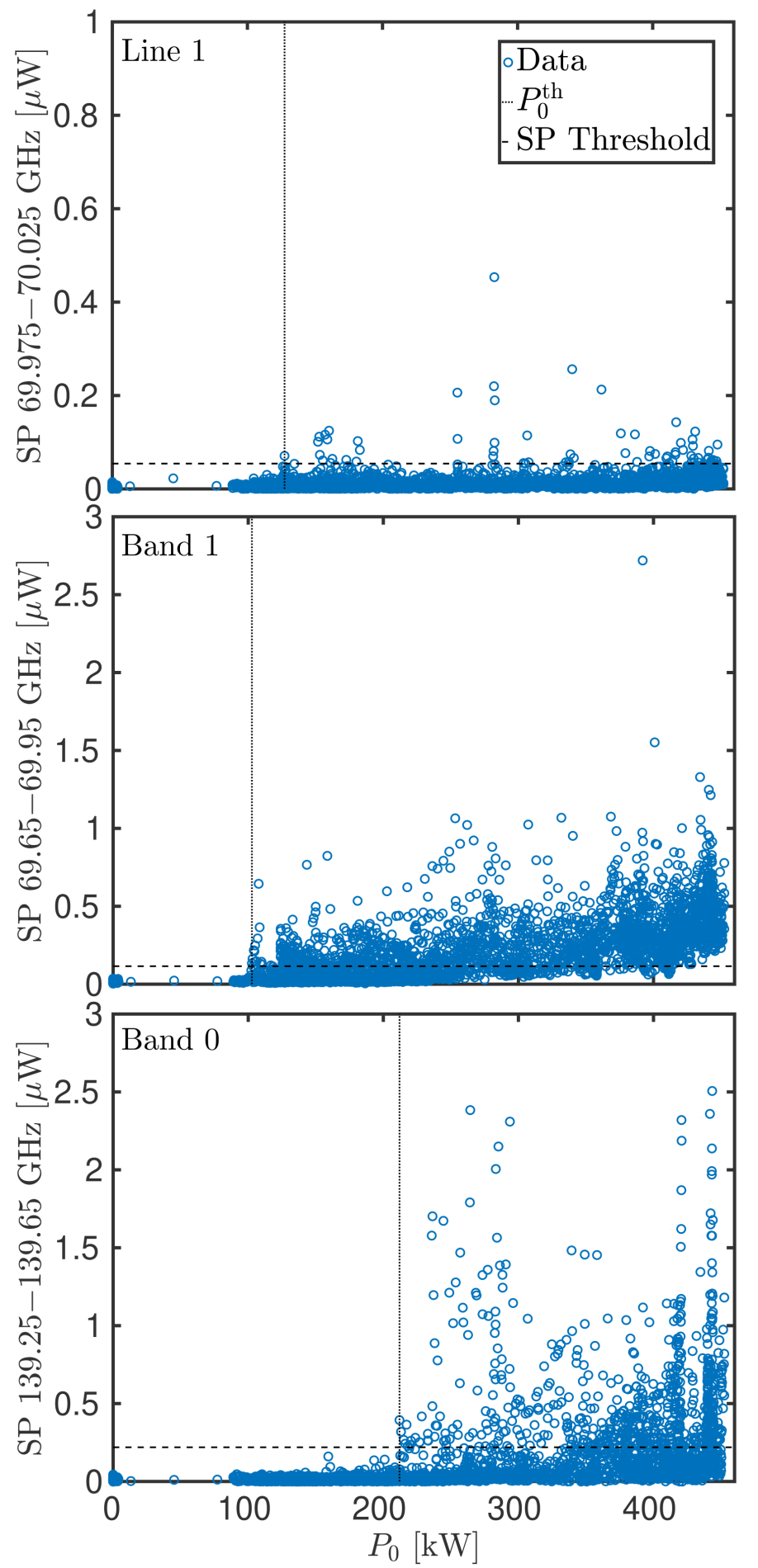

Figure 10. SP in the frequency ranges of line $1(69.975-70.025 \mathrm{GHz})$, band 1 $(69.65-69.95 \mathrm{GHz})$, and band $0(139.25-139.65 \mathrm{GHz})$ from the fast-acquisition CTS system versus $P_{0}$ just after the probe gyrotron is switched on in Discharge 2 . We compute $P_{0}^{\text {th }}$ (fine dotted lines) as the lowest $P_{0}$ values for which SP thresholds (dashed lines) of $20 \mathrm{SP}$ standard deviations above the mean SP before the probe gyrotron is switched on are exceeded. 


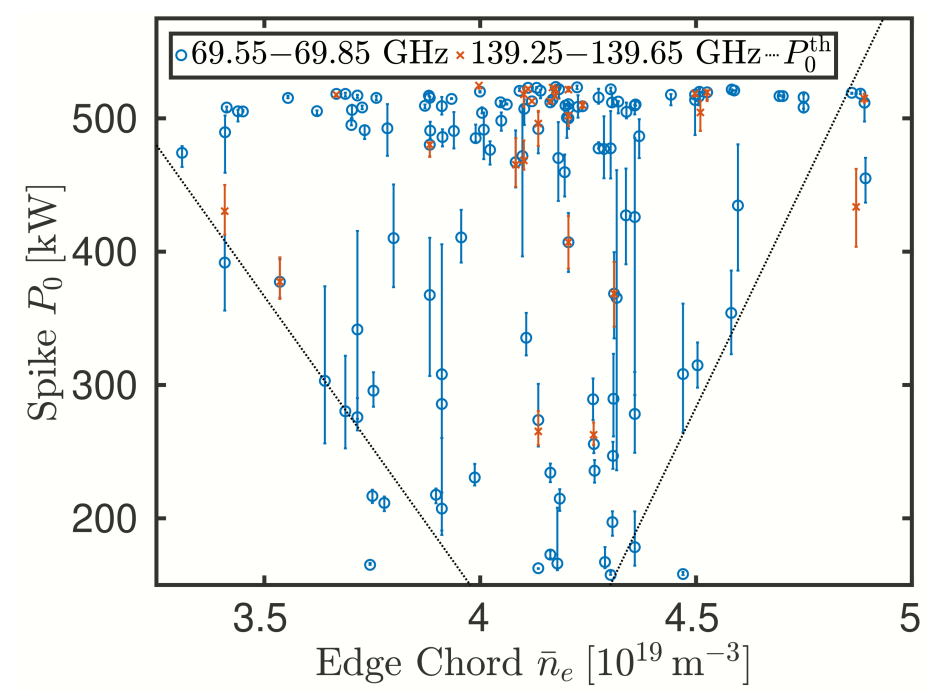

Figure 11. Values of $P_{0}$ during the microwave spikes in the frequency intervals 69.55-69.85 GHz (o) and 139.25-139.65 GHz (×) of Discharge 2 versus $\bar{n}_{e}$ from the edge chord; the errorbars are determined by the $P_{0}$ variation over the duration of the individual spikes. At a given $\bar{n}_{e}$, spikes only appear above a particular $P_{0}$, which we identify as $P_{0}^{\text {th }}$. The dash-dotted lines show empirical estimates of $P_{0}^{\text {th }}$ in the low and high $\bar{n}_{e}$ ranges, calculated using the method outlined in the text.

the algorithm employed in constructing figure 7 and obtain the $P_{0}$ at which each spike occurs by interpolating the $P_{0}$ signal to the spike time points identified by the algorithm; errorbars of $P_{0}$ are further obtained through the $P_{0}$ variation over the duration of the individual spikes. When the $P_{0}$ values of the spikes in the frequency ranges $69.55-69.85$ $\mathrm{GHz}$ and $139.25-139.65 \mathrm{GHz}$ are plotted against $\bar{n}_{e}$ from the edge chord (H-5), figure 11 emerges. Figure 11 shows the strong correlation of the spikes near $140 \mathrm{GHz}$ with the spikes near $70 \mathrm{GHz}$ expected from figure 8, as well as the previously mentioned higher $P_{0}$ necessary for the occurrence of the spikes near $140 \mathrm{GHz}$ compared with those near $70 \mathrm{GHz}$, consistent with them originating from a higher-order PDI [10]. Additionally, the spikes in both frequency ranges display a distinct $P_{0}$ value which must be exceeded in order for spikes to occur at a given edge chord $\bar{n}_{e}$, which we identify as $P_{0}^{\text {th }}$. The $P_{0}^{\text {th }}$ values in both frequency ranges appear to decrease with increasing $\bar{n}_{e}$ for low edge chord $\bar{n}_{e}$ values $\left(3.3-4.0 \times 10^{19} \mathrm{~m}^{-3}\right)$, reach a minimum at intermediate values $\left(4.0-4.3 \times 10^{19} \mathrm{~m}^{-3}\right)$, and increase for larger values $\left(4.3-5.0 \times 10^{19} \mathrm{~m}^{-3}\right)$. To obtain an empirical estimate of the primary $P_{0}^{\text {th }}$ in the high and low $\bar{n}_{e}$ ranges, which may be compared with theory, we least-square fit a linear function of $\bar{n}_{e}$ to the minimum $P_{0}$ values of the spikes in the frequency range $69.55-69.85 \mathrm{GHz}$ for bins with widths of $10^{18} \mathrm{~m}^{-3}$ in each of these $\bar{n}_{e}$ ranges; the intermediate $\bar{n}_{e}$ values are not treated, as the primary $P_{0}^{\text {th }}$ for these values may be below the lowest $P_{0}$ during the analog $P_{0}$ modulations $(150 \mathrm{~kW})$. The resulting fits of the primary $P_{0}^{\text {th }}$ are seen in figure 11 and show good agreement with the $P_{0}$ edge above which spikes occur. For a comparison with the JOREK simulation of an ELM crash in Discharge 4, we note that the edge 
chord $\bar{n}_{e}=3.76 \times 10^{19} \mathrm{~m}^{-3}$ for the time point of Discharge 4 shown in figure 3, which serves as an initial condition for the ELM simulation, giving an empirical estimate of $P_{0}^{\text {th }}=250 \mathrm{~kW}$ to be compared with the value obtained by applying the theory of section 2 to the JOREK simulation. Finally, we note that the possibility of computing $P_{0}^{\text {th }}$ based on the strong relation between the $P_{0}$ value at which spikes appear and the edge chord $\bar{n}_{e}$, rather than $\bar{n}_{e}$ from a central chord, is a strong indication that the signals originate from the plasma edge during ELMs, as further demonstrated in the discussion of Discharge 3.

\section{Discharge 3: Source Location and ICRH Impact}

Before discussing the JOREK simulation of Discharge 4, we address the issues of the spatial location of the source of the spikes near $140 \mathrm{GHz}$ and the impact of ICRH on the spike duration distribution in Discharge 3.

The location of the origin of the strong signal near $140 \mathrm{GHz}$ is pin-pointed by sweeps of the CTS receiver view along the probe gyrotron beam, performed by scanning the position of the spindle of the CTS reveiver mirror during the discharge [7]. Figure 12 shows the SP from $139.25-139.65 \mathrm{GHz}$ versus $R_{\text {overlap }}$, defined as the $R$ value at which the central ray of the receiver view is closest to the central ray of the probe gyrotron beam; cf. figure 3, where $R_{\text {overlap }}=1.989 \mathrm{~m}$ for the time point shown for Discharge 3 . Evidently, the large microwave spikes just below the ECRH frequency associated with ELMs only occur when $R_{\text {overlap }}$ is sufficiently large, corresponding to a receiver view overlapping with the probe gyrotron near the plasma edge, which is illustrated by the location of the last closed flux surface in figure 12. This is a clear indication that the signal originates from the probe gyrotron beam near the plasma edge, as expected if its source is PDIs in the ELM filaments.

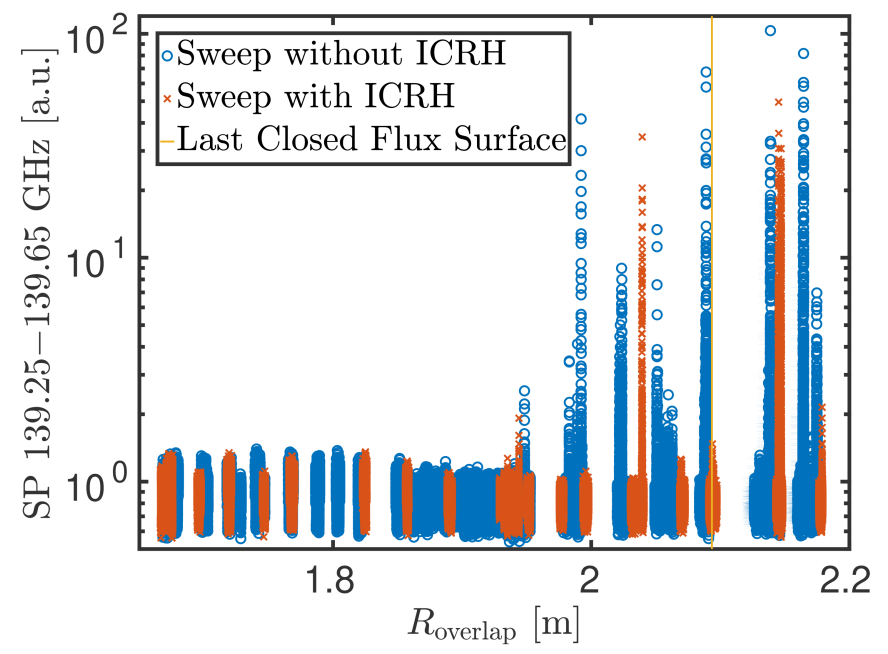

Figure 12. SP in the range 139.25-139.65 GHz from the fast-acquisition CTS system versus $R_{\text {overlap }}$ in Discharge 3. Strong signals are observed for overlaps near the plasma edge, as indicated by the solid line giving the location of the last closed flux surface. 

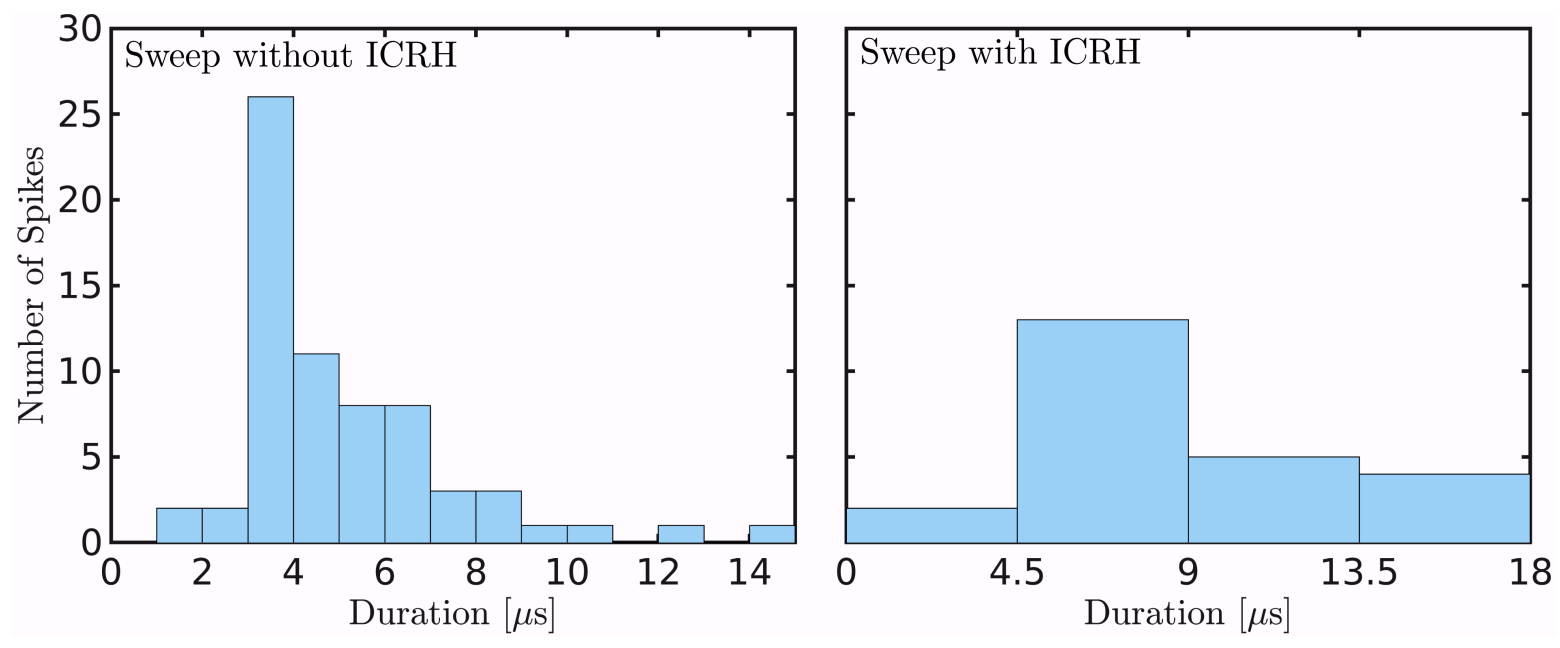

Figure 13. Histograms of the microwave spike duration in the frequency range 139.25-139.65 GHz during the receiver sweeps without and with ICRH in Discharge 3. The spike duration is generally longer during the sweep with ICRH.

As indicated in figure 12, two sweeps of the receiver view are performed, one without ICRH of the plasma and one with (1.8 MW) ICRH of the plasma. Histograms of the duration of the spikes from $139.25-139.65 \mathrm{GHz}$ in the two sweeps, calculated using the same method as in figure 7, are shown in figure 13. For the sweep without ICRH, the spike duration distribution is relatively similar to that of Discharge 1, seen in figure 7, albeit with a somewhat increased general spike duration, illustrated by the mean of $5.08 \pm 0.29 \mu \mathrm{s}$, the standard deviation of $2.41 \pm 0.21 \mu \mathrm{s}$, and the maximum probability density from $3-4 \mu \mathrm{s}$. For the sweep with ICRH, the general spike duration is significantly increased, indicated by the mean now being $8.7 \pm 0.9 \mu \mathrm{s}$, the standard deviation being $4.3 \pm 0.6 \mu \mathrm{s}$, and the maximum probability density from $4.5-9 \mu \mathrm{s}$; the distribution still appears to have a shape similar to that in the earlier case, though the small number of observed spikes makes this somewhat uncertain. The difference of the spike duration distributions for the two sweeps may be explained by the fact that ICRH changes the poloidal drift velocities of the ELM filaments through a steady radial electric field induced by sheath rectification of the injected radiation [56 58].

\section{Discharge 4: Comparison with JOREK Simulation}

Having discussed the experimental observations, we now turn to the issue of comparing them with the theoretical predictions of section 2. In order to apply the theory, detailed knowledge of $n_{e}$ and $T_{e}$ at the plasma edge is required with a time resolution $\sim 1 \mu \mathrm{s}$. Since such information is generally not available experimentally, the required information is extracted from a simulation of an ASDEX Upgrade discharge using the nonlinear MHD code JOREK 34 36]. Specifically, we look at the simulation of an ELM crash from Discharge 4, described in [35, 36]; $B$ is extracted from the CLISTE equilibrium [50] used as the initial condition for the simulation, shown in figure 3, and 
is assumed to remain virtually constant during the ELM. We estimate $P_{0}^{\text {th }}$ along with the burst duration using the theory of section 2, As seen in figure 3, Discharge 4 has a similar plasma shape to Discharges 1 and 2; table 1 further shows that the $I_{p}, B_{t}$, and heating mix of Discharges 1 and 2 have been chosen to match Discharge 4 , leading to similar $n_{e}$ and $T_{e}$ profiles before the start of the $n_{e}$ scans.

The spike duration distributions found in figure 7 are consistent with the passage time of the $n_{e}$ structures allowing trapping during an ELM crash through an ECRH beam. This is demonstrated in figure 14, which shows the evolution of $n_{e}$ and the warm $70 \mathrm{GHz}$ UHR according to the JOREK simulation of Discharge 4 [35,36]. For the snapshots shown in figure 14, the simulation time, $t$ (which is 0 at the beginning of the ELM crash), runs from $52.1-60.5 \mu \mathrm{s}$ and during this time interval $(8.4 \mu \mathrm{s})$, a region allowing trapping of two $70 \mathrm{GHz} \mathrm{UH}$ plasmons, which is necessary for the considered PDIs to generate microwave spikes, passes through the lower pair of ECRH beams. The time interval of $8.4 \mu \mathrm{s}$ thus represents an (upper) estimate of the spike duration which is similar to the experimental values from figure 7. The agreement with experiment is only obtained due to the fact that the JOREK simulation is based on extended MHD, which takes neoclassical and diamagnetic drift effects into account, such that the plasma flows are obtained self-consistently. This causes the radial electric field to evolve in a manner comparable to the experimental observations during the ELM crash, as shown in [35], which is essential not only for capturing the ELM dynamics correctly, but also for obtaining correct transit times of the non-monotonic $n_{e}$ structures across the ECRH beams.

We can further use the theory developed in section 2 to estimate $P_{0}^{\text {th }}$ when the trapping region crosses the central rays of the ECRH beams; the plasma and beam parameters used for these calculations are extracted along the lower pair of ECRH beams in figure 14 at $t=55.4 \mu \mathrm{s}$ and shown in figure 15 . For pane (a) of figure 15 the resulting profiles give the parameters $m=5, k_{y}=3.78 \mathrm{~mm}^{-1}, v_{y}=264 \mathrm{~km} / \mathrm{s}$, $\Gamma=141 \mathrm{~ms}^{-1}$, and $\sqrt{\gamma_{12}^{\text {ref }}(0,0) \gamma_{21}^{\text {ref* }}(0,0)}=19.9 \mu \mathrm{s}^{-1}$ at $P_{0}^{\text {ref }}=500 \mathrm{~kW}$; for pane (b) of figure 15 the parameters are $m=8, k_{y}=2.89 \mathrm{~mm}^{-1}, v_{y}=258 \mathrm{~km} / \mathrm{s}, \Gamma=140 \mathrm{~ms}^{-1}$, and $\sqrt{\gamma_{12}^{\text {ref }}(0,0) \gamma_{21}^{\text {ref } *}(0,0)}=19.7 \mu \mathrm{s}^{-1}$ at $P_{0}^{\text {ref }}=500 \mathrm{~kW}$. Following $[6]$, we set $L_{y}=\sqrt{\pi / 8} W$, where $W=2.873 \mathrm{~cm}$ is the (1/e electric field) width of the Gaussian ECRH beams in the trapping regions, and with this we obtain $P_{0}^{\text {th }}=175 \mathrm{~kW}$ and $P_{0}^{\text {th }}=171 \mathrm{~kW}$ for panes (a) and (b) of figure 15, respectively; the relative error of the analytical approximation of $P_{0}^{\text {th }}$ from 15 compared with the numerical solution of 12 is $<7.6 \times 10^{-7}$ in both cases. Use of the estimates from [5] give significantly lower values of $P_{0}^{\text {th }}, 2.65 \mathrm{~kW}$ and 2.63 $\mathrm{kW}$ for panes (a) and (b) of figure 15, respectively, with the threshold due to diffraction losses along $\mathbf{B}$ being higher than that due to convection losses, despite the domination of the convection loss terms; the reason for these issues are outlined in $[6]$. The $P_{0}^{\text {th }}$ values of $171-175 \mathrm{~kW}$ are similar to the experimentally estimated $P_{0}^{\text {th }}$ in Discharge 2 (250 kW, based on figure 11), providing further evidence in favor of the second-harmonic UHR PDI theory of the microwave spikes. Considering the greatly simplified theoretical model used for determining $P_{0}^{\text {th }}$ and the MHD nature of the simulation, the agreement 


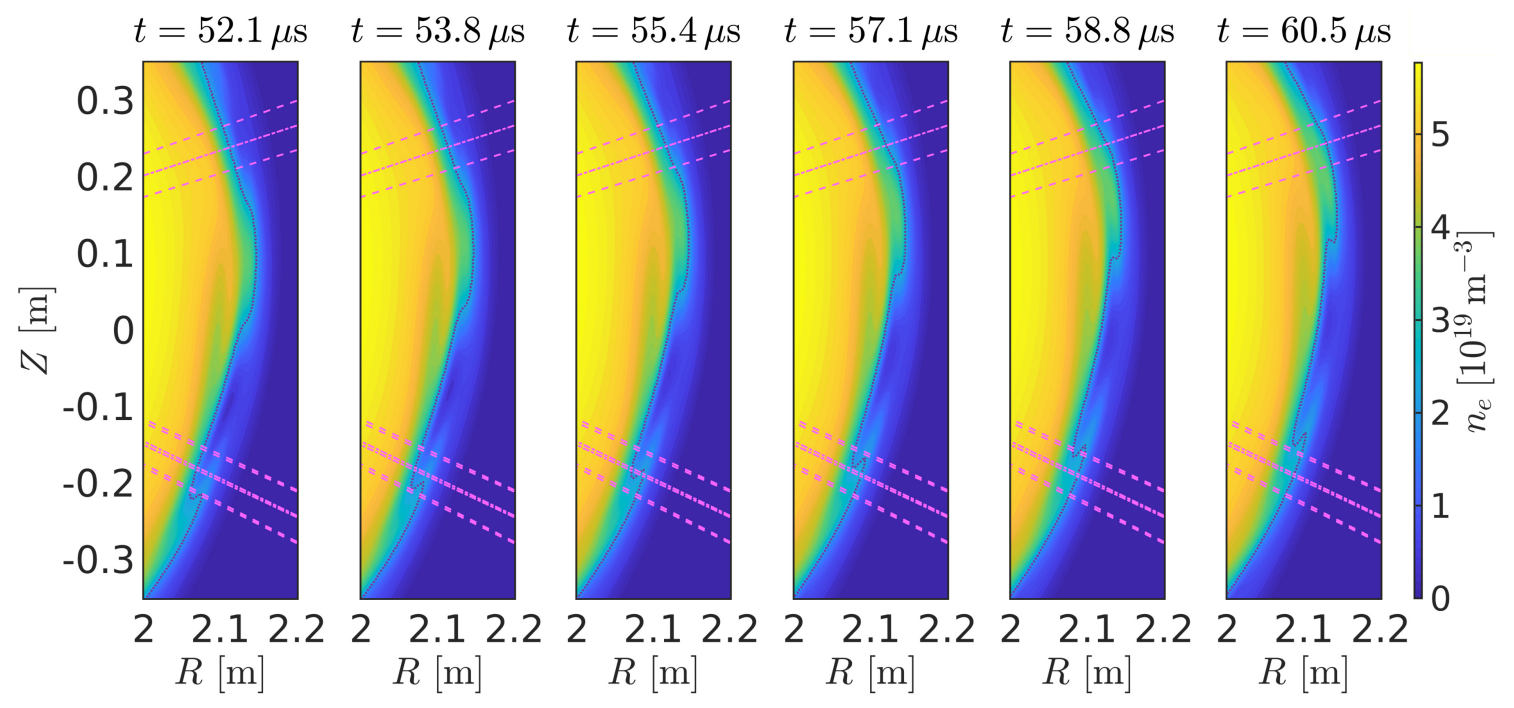

Figure 14. Evolution of $n_{e}$ and the warm $70 \mathrm{GHz}$ UHR (fine dotted line) in the JOREK simulation of Discharge 4. The dashed-dotted lines mark the gyrotron central beam rays; the dashed lines mark the (1/e electric field) widths of the Gaussian ECRH beams.
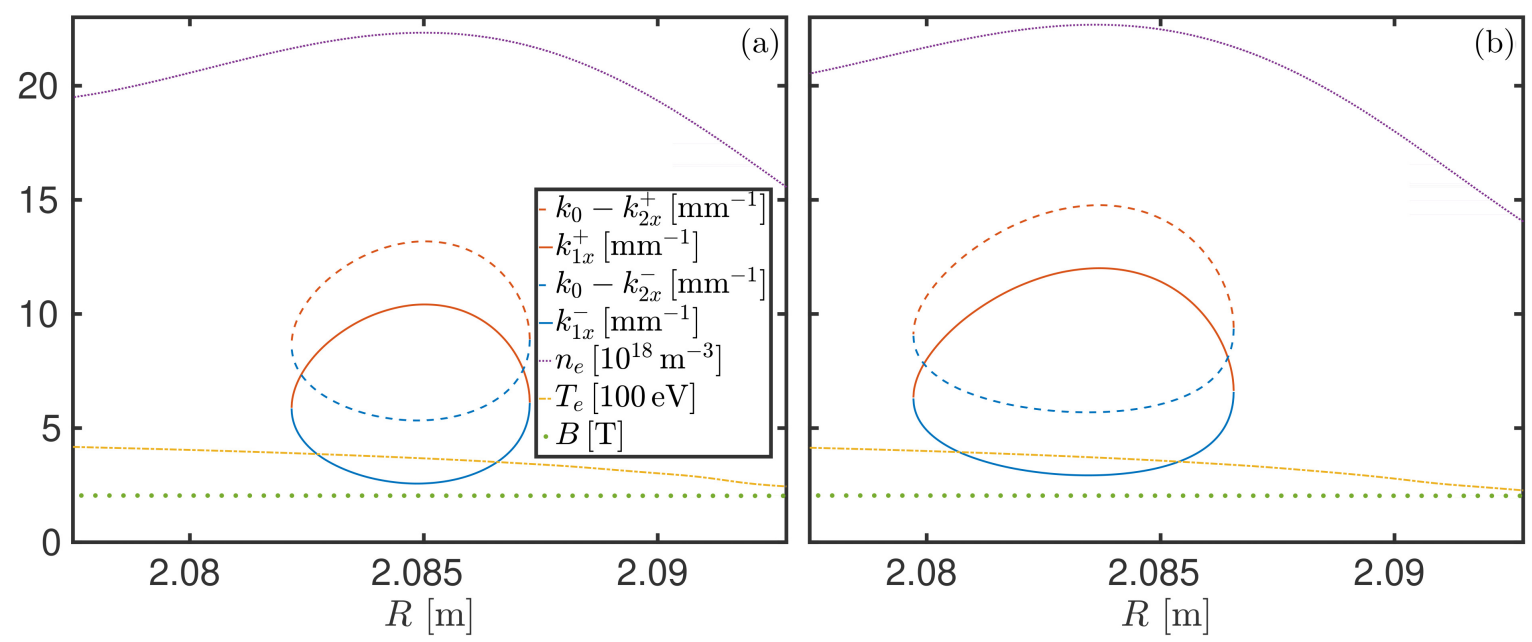

Figure 15. Plasma and wave parameters in the trapping regions of the lower beams at $t=55.4 \mu \mathrm{s}$ in the JOREK simulation of Discharge 4; see figure 14. Pane (a) shows the situation for the beam with $P_{0}^{\text {th }}=175 \mathrm{~kW}$; pane (b) shows the situation for the beam with $P_{0}^{\text {th }}=171 \mathrm{~kW}$.

is very good.

\section{Discussion}

With the experimental and numerical results presented, we are now in a position to discuss the interpretation of the obtained results, particularly the nature of the signals with frequencies near half the ECRH frequency reaching the CTS receiver.

As seen in panes (a) and $\left(\mathrm{a}^{*}\right)$ of figure 9, the PDI signals during the L-mode phase 
consist of a narrow line at exactly half the ECRH frequency (line 1), as well as broadband features, such as band 1. On the other hand, the PDI signals during the H-mode phase, seen in panes (1a) and (1b) of figure 6, consist of a number of bands, significantly wider than line 1, during the inter-ELM phases, augmented by broadband spikes during the ELMs. We further note that the microwave signals near half the ECRH frequency are not limited to a narrow $n_{e}$ range, since they appear throughout the $n_{e}$ scan shown in figure 5 with only minor changes. Additionally, microwave signals are present above, as well as below, half the ECRH frequency. The above features are at odds with the mechanism of microwave emissions near half the ECRH frequency proposed in [13, where it is assumed that the primary daughter wave with a frequency below half the ECRH frequency is non-trapped (limiting its occurrence to a narrow $n_{e}$ range), such that its X-mode branch can escape the plasma on the high-field side, be cross-polarized to O-mode by a reflection at the high-field side vessel wall, and then reach the receiver at the low-field side of the plasma, provided that absorption at the ECR is limited. We hence consider alternative mechanisms allowing emission of microwaves near half the ECRH frequency.

An obvious candidate is direct tunneling of the X-mode branch of the trapped daughter waves through the evanescence layer between the trapping region and the lowfield side of the tokamak. To estimate the strength of this signal for wave $j$, we utilize the Budden power transmission coefficient, $T_{j}$, from cold plasma theory 61, 62,

$$
T_{j}=\mathrm{e}^{-\pi d_{j} \omega_{j} / c},
$$

where $d_{j}$ is the width of the evanescence region of wave $j$. For the JOREK simulation of Discharge 4, the narrowest width of the evanescence layer around the trapping region is $d_{j} \approx 7 \mathrm{~mm}$, leading to $T_{j} \lesssim 10^{-14}$. Thus, even assuming that the daughter waves carry the maximum power of the pump wave (approximately $500 \mathrm{~kW}$ ), the transmitted power will be $\lesssim 5 \mathrm{nW}$, which is far below the SP recorded by the $70 \mathrm{GHz}$ system during an ELM, cf. figure 8. We note that use of the Budden power transmission coefficient does not account for modifications of the transmission through the X-mode evanescence layer due to standing wave effects, but is appropriate if we treat the cavity as a slab with a transmission (and reflection) coefficient associated with each interface, since the referenced power is that circulating in the cavity [63]. If we instead were to use the mode coupling coefficient commonly used in connection with X-B heating and B-X emission in spherical tokamaks 3841 , we would find $T_{j}<4 \mathrm{e}^{-\pi d_{j} \omega_{j} / c}$, leading to a transmitted power $\lesssim 20 \mathrm{nW}$, which is still far below the SP recorded by the $70 \mathrm{GHz}$ system during an ELM, cf. figure 8. The mode coupling coefficients of 38 41] include the effect of X-mode waves being reflected toward the UHR by an L-cutoff, which can be treated using the cold plasma approximation. By contrast, the cavity effect of relevance in the present paper is mode conversion of X-mode waves to EBWs (and vice versa) at the UHR near the main plasma and their return to the UHR near the vessel wall, which would necessitate the inclusion of hot plasma effects to be properly accounted for. Based on the above analysis, direct tunneling is not able to account for the strong signals 
near half the ECRH frequency from H-mode plasmas. However, L-mode blobs have a significantly smaller spatial extent than ELM filaments, compare figure 14 with figure 2 of [24], meaning that direct tunneling may account for some strong signals from L-mode plasmas. Particularly, the narrow line at exactly half the ECRH frequency seems to originate from direct tunneling of the primary daughter waves through the evanescence region, as it disappears during the $\mathrm{H}$-mode phase and is not broadened by secondary nonlinear wave interactions.

The strong signals near half the ECRH frequency during the H-mode phase, as well as the broadband features during the L-mode phase, seem to require conversion of the trapped EBWs/X-mode waves to O-mode waves (cross-polarization) for their power to be able to reach the CTS receiver, since this mechanism is insensitive to the exact width of the $\mathrm{X}$-mode evanescence layer and is not limited to a narrow $n_{e}$ range. Cross-polarization is a three-wave effect facilitated by mixing of the waves near half the ECRH frequency with low-frequency waves and PDIs involving low-frequency waves and waves near half the ECRH frequency [64]. The requirement of interactions with low-frequency waves leads to the observed frequency shifts and broadening of the microwave bands relative to the direct tunneling line during the L-mode phase, due to the three-wave interaction frequency selection rule. Additionally, this mechanism explains why the bands near $70 \mathrm{GHz}$, which are related to the primary daughter waves, are weaker than the bands possessing frequency shifts near multiples of 880 $\mathrm{MHz}$ from these bands in figure 5 during the inter-ELM phases: waves near $880 \mathrm{MHz}$ are abundant due to the occurrence of secondary PDIs, allowing generation of significant O-mode power at frequencies shifted by multiples of $880 \mathrm{MHz}$ from the primary daughter waves, while the waves near $70 \mathrm{GHz}$ can only be converted to O-mode through mixing with low-frequency fluctuations. The particular weakening of the bands near $70 \mathrm{GHz}$ during ELMs observed in figure 6 can be interpreted as being due to the altered lowfrequency fluctuations during ELMs within this framework, while the broadband spikes observed during ELMs are attributed to the different trapping characteristics of the ELM filaments compared with the inter-ELM modes [23] that generate the narrower bands discussed above. The broadband signals observed during the L-mode phase appear to be similar to the broadband spikes related to ELMs and are interpreted as cross-polarized radiation generated by PDIs in the L-mode blobs.

We interpret the strong signals near the ECRH frequency in the same way as [10], namely as (X-mode) microwaves generated by combination of two daughter waves near half the ECRH frequency. In connection with ELMs and L-mode blobs, these signals appear as broadband spikes coinciding with the spikes near half the ECRH frequency, while a narrow band down-shifted by approximately $1.5 \mathrm{GHz}$ from the ECRH frequency is present during the inter-ELM phase at low $n_{e}$. The latter signal is consistent with combination of waves from two of the bands near half the ECRH frequency (particularly, bands 1 and 3 of figure 5). The disappearance of the narrow band during the inter-ELM phase at higher $n_{e}$ is consistent with the weakening of the bands near half the ECRH frequency observed in figure 5 and with the fact that they are a consequence of a 
higher-order PDI. We further note that the fact that not all of the bands present near half the ECRH frequency are recovered near the ECRH frequency is likely related to the impossibility of satisfying the wave vector selection rules when combining certain modes to a microwave near twice their frequency. Finally, the fact that strong signals occur almost exclusively below the ECRH frequency is a strong indication that they are a result of PDIs driven by the ECRH radiation.

There are several points deserving further theoretical, numerical, and experimental investigations. First, the interpretation of the signals near half the ECRH frequency as originating from cross-polarized trapped waves should be investigated analytically. According to [64], the cross-polarized signals near the UHR originate from (electromagnetic) fluctuations of $\mathbf{B}$, as well as from fluctuations of the electron velocity parallel to the background $\mathbf{B}$. Neither of these effects are included in the basic theory of PDIs near the second-harmonic UHR in a plasma slab, since it assumes electrostatic trapped daughter waves propagating perpendicular to the background magnetic field. An inclusion of electromagnetic effects and oblique propagation of the daughter waves may thus be necessary to estimate the theoretically expected signal levels near half the ECRH frequency, which could be compared with the experimental observations of the present paper; we note that oblique daughter wave propagation has already been included in the theory of PDIs near the second-harmonic UHR in (circular) plasma filaments 11, 16, 17]. Further, the applicability of the PDI theory to the signals observed in connection with L-mode turbulence should be confirmed in a similar manner to that of the ELM-related spikes, using the output of an edge turbulence code, e.g., HESEL [24] or GRILLIX [65], which could also be used to confirm the possibility of direct tunneling of the half-frequency X-mode daughter waves through the cutoff region. The character of the signals should additionally be investigated experimentally (and numerically) in a wider range of scenarios than just L-modes developing into type I ELMy H-modes. For instance, it would be of interest to characterize the signals in I-mode plasmas, particularly looking for quasi-continuous signals related to the weakly coherent mode and spikes related to I-mode bursts [66]. Other interesting regimes are H-mode plasmas with small ELMs [67] and ELM-free H-modes, such as the QH-mode [68]. Finally, the relation of the PDI microwave signals to other strong microwave signals generated by ECE from fast electrons is of interest. We particularly note that the narrowband bursts observed in connection with the edge harmonic oscillation in QH-mode plasmas and attributed to ECE associated with fast electrons [69] may have a PDI component as well.

\section{Conclusion}

At the magnetic field strengths used for central second-harmonic X-mode ECRH in present-day conventional tokamaks, the second-harmonic UHR of ECRH radiation occurs at low electron densities, meaning that it will usually appear in the edge region. This allows trapping of UH daughter waves near half the ECRH frequency in relatively 
small edge density perturbations, associated with ELMs, inter-ELM modes, and L-mode blobs, leading to a low-threshold PDI in which such waves are excited by the ECRH radiation [5,6,10 15]. These PDIs may be detected by a radiometer observing an ECRH beam near the plasma edge; in this paper, we have specifically used an upgraded version of the CTS system at the ASDEX Upgrade tokamak, with a filter bank and a fastacquisition system operating near half the ECRH frequency $(70 \mathrm{GHz})$ in addition to the original systems operating near the ECRH frequency itself (140 GHz). The upgraded CTS system permitted the first investigation of the signals associated with PDIs near half the ECRH frequency under fusion-relevant conditions, as well as the relation of these signals to the previously-observed signals near the ECRH frequency. The signals in both frequency regions have a different character in L-mode and H-mode plasmas.

For L-mode plasmas, relatively broadband quasi-continuous, strong signals are present below the ECRH frequency and around half the ECRH frequency, along with a narrow quasi-continuous line very close to half the ECRH frequency. All signals can be attributed to PDIs occurring in the blobs which are continually ejected from Lmode plasmas [24]. By performing modulations of the power of the ECRH gyrotron overlapping with the CTS receiver view near the plasma edge, we confirmed that the signals near half the ECRH frequency have similar PDI power thresholds, while the signals near the ECRH frequency have a significantly higher PDI power threshold. These observations are consistent with the theoretical expectation that the signals near the ECRH frequency originate from a higher-order PDI than the ones near half the ECRH frequency [10] and further indicate that the signals originate from the modulated gyrotron beam.

For H-mode plasmas, the signals during inter-ELM and ELM phases are distinct. During inter-ELM phases, the signals near half the ECRH frequency consist of distinct quasi-continuous frequency bands, specifically two bands, one slightly above and one slightly below half the ECRH frequency, along with a number of bands up- and downshifted by multiples of $880 \mathrm{MHz}$ from one of these bands, which may be attributed to secondary PDIs [10]. Near the ECRH frequency, a band corresponding to combination of the band slightly above half the ECRH frequency with the most down-shifted band is observed at low electron densities, consistent with the fact that the bands around half the ECRH frequency are strongest for lower electron densities and with the signals near the ECRH frequency originating from a higher-order PDI. During ELMs, the quasicontinuous lines are (partially) replaced by broadband microwave spikes, indicating alteration of the wave trapping regions near the plasma edge. The duration of the microwave spikes observed near the ECRH frequency and half the ECRH frequency with the fast-acquisition CTS system is in agreement with the passage time of the regions allowing daughter wave trapping through an ECRH beam according to a nonlinear MHD simulation of an ELM crash, performed using the JOREK code [34, 36]. Additionally, the PDI thresholds obtained from an analytical model using input from the JOREK simulation agree well with the PDI thresholds inferred from the lowest power at which spikes occur during modulations of the power of the ECRH beam overlapping with 
the CTS receiver view near the plasma edge. The experimental PDI thresholds near the ECRH frequency are further found to be higher than those near half the ECRH frequency, consistent with them originating from a higher-order PDI, as claimed earlier. The spike duration is also seen to be strongly influenced by the inclusion of ICRH, which may be attributed to an alteration of the poloidal velocity of the ELM filaments due to sheath rectification of the ICRH radiation in the scrape-off layer [56 58. Finally, the spikes during ELMs are seen to have a vastly increased amplitude for CTS receiver views overlapping with an ECRH gyrotron near the plasma edge, supporting their PDI-origin.

The PDI-related microwave signals observed in connection with ELMs, inter-ELM modes, and blobs are generally not at a level which poses any danger to microwave diagnostics, and in order to reliably observe them, it is necessary for the view of a radiometer to overlap with an ECRH beam near the plasma edge. They do, however, present the possibility of creating a new type of edge diagnostic which is able to measure the passage of ELM filaments, changes in the character of the edge turbulence, and interELM modes in the localized region of an ECRH beam with high temporal resolution.

\section{Acknowledgements}

We thank Prof. E. Poli for providing valuable feedback on an early version of this paper and Prof. E. Z. Gusakov for directing our attention toward the work of [43]. SKH acknowledges support by an Internationalisation Fellowship (CF19-0738) from the Carlsberg Foundation. This work was supported by a research grant (15483) from VILLUM FONDEN. This work has been carried out within the framework of the EUROfusion Consortium and has received funding from the Euratom research and training programme 2014-2018 and 2019-2020 under grant agreement No 633053. The views and opinions expressed herein do not necessarily reflect those of the European Commission.

\section{References}

[1] Piliya A D 1971 Decay instability in weakly inhomogeneous plasma Proc. 10th Int. Conf. Phenomena in Ionized Gases (Oxford) ed Franklin R N (Oxford: Donald Parsons \& Co. Ltd.) p 320

[2] Rosenbluth M N 1972 Phys. Rev. Lett. 29 565-567

[3] Piliya A D 1973 Sov. Phys.-JETP 37 629-632 URL http://www.jetp.ac.ru/cgi-bin/dn/e_ 037_04_0629.pdf

[4] Porkolab M and Cohen B I 1988 Nucl. Fusion 28 239-254

[5] Popov A Yu and Gusakov E Z 2015 Plasma Phys. Control. Fusion 57025022

[6] Hansen S K 2019 Parametric Decay Instabilities in the Electron Cyclotron Resonance Heating Beams at ASDEX Upgrade Ph.D. thesis Technical University of Denmark Kgs. Lyngby

[7] Wagner D, Grünwald G, Leuterer F, Manini A, Monaco F, Münich M, Schütz H, Stober J, Zohm H, Franke T, Thumm M, Gantenbein G, Heidinger R, Meier A, Kasparek W, Lechte C, Litvak A, Denisov G G, Chirkov A V, Tai E M, Popov L G, Nichiporenko V O, Myasnikov V E, Solyanova E A, Malygin S A, Meo F and Woskov P 2008 Nucl. Fusion 48054006 
[8] Stober J, Reisner M, Angioni C, Bañón Navarro A, Bobkov V, Bock A, Denisov G, Fable E, Fischer R, Gantenbein G, Gil L, Görler T, Igochine V, Kasparek W, Leuterer F, Litvak A, McDermott R, Meier A, Monaco F, Münich M, Nichiporenko V, Plaum B, Plank U, Poli E, Popov L, Pütterich Th, Scherer Th, Schubert M, Suttrop W, Tai E, Thumm M, Wagner D, Zohm H, MST1 team and the ASDEX Upgrade team 2020 Plasma Phys. Control. Fusion 62024012

[9] Gusakov E Z and Fedorov V I 1979 Sov. J. Plasma Phys. 5 463-466

[10] Gusakov E Z and Popov A Yu 2016 Phys. Plasmas 23082503

[11] Gusakov E, Popov A and Sysoeva E 2017 EPJ Web Conf. 15703019

[12] Gusakov E Z and Popov A Yu 2018 Plasma Phys. Control. Fusion 60025001

[13] Gusakov E Z, Popov A Yu and Tretinnikov P V 2019 Nucl. Fusion 59106040

[14] Gusakov E Z and Popov A Yu 2019 Nucl. Fusion 59104003

[15] Gusakov E Z and Popov A Yu 2020 Plasma Phys. Control. Fusion 62025028

[16] Simonchik L V, Altukhov A B, Arkhipenko V I, Gurchenko A D, Gusakov E Z, Popov A Y and Usachonak M S 2017 EPJ Web Conf. 14903013

[17] Altukhov A B, Arkhipenko V I, Gurchenko A D, Gusakov E Z, Popov A Yu, Simonchik L V and Usachonak M S 2019 EPL 12615002

[18] Westerhof E, Nielsen S K, Oosterbeek J W, Salewski M, De Baar M R, Bongers W A, Bürger A, Hennen B A, Korsholm S B, Leipold F, Moseev D, Stejner M, Thoen D J and the TEXTOR team 2009 Phys. Rev. Lett. 103125001

[19] Nielsen S K, Salewski M, Westerhof E, Bongers W, Korsholm S B, Leipold F, Oosterbeek J W, Moseev D, Stejner M and the TEXTOR team 2013 Plasma Phys. Control. Fusion 55115003

[20] Nielsen S K, Jacobsen A, Kjer Hansen S, Korsholm S B, Leipold F, Rasmussen J, Salewski M, Stejner M, Stober J, Scubert M, Wagner D and the ASDEX Upgrade team 2016 Three-wave interaction during electron cyclotron resonance heating and current drive Proc. 41st Int. Conf. Infrared, Millimeter, and Terahertz Waves (Copenhagen) (Piscataway: IEEE)

[21] Hansen S K, Nielsen S K, Stober J, Rasmussen J, Salewski M, Stejner M, Hoelzl M and the ASDEX Upgrade Team 2019 Europhys. Conf. Abstracts 43C P1.1075 URL http://ocs.ciemat.es/ EPS2019PAP/pdf/P1.1075.pdf

[22] Senstius M G, Nielsen S K and Vann R G L 2020 Phys. Plasmas 27062102

[23] Vanovac B, Denk S S, Wolfrum E, Willensdorfer M, Suttrop W, Fischer R, Luhmann Jr N C and the ASDEX Upgrade Team 2019 EPJ Web Conf. 20302011

[24] Nielsen A H, Juul Rasmussen J, Madsen J, Xu G S, Naulin V, Olsen J M B, Løiten M, Hansen S K, Yan N, Tophøj L and Wan B N 2017 Plasma Phys. Control. Fusion 59025012

[25] Porte L, Bartlett D V, Campbell D J and Costley A E 1991 Europhys. Conf. Abstracts 15C IV 357-360

[26] Taylor G, Bush C E, Fredrickson E D, Park H K and Ramsey A T 1992 Nucl. Fusion 32 1867-1872

[27] Bartlett D V, Costley A E, Jones S E, Porte L, Smith R J and Zolfaghari A 1993 The JET ECE heterodyne radiometer and investigations of fast phenomena Proc. 8th Joint Workshop ECE and ECRH (Ising) (Garching: Max-Planck-Institut für Plasmaphysik) pp 251-263

[28] Janos A, Hastie J, McGuire K and Fredrickson E 1996 Plasma Phys. Control. Fusion 38 1373-1379

[29] Fuchs Ch and Austin M E 2001 Phys. Plasmas 8 1594-1599

[30] Meo F, Stejner M, Salewski M, Bindslev H, Eich T, Furtula V, Korsholm S B, Leuterer F, Leipold F, Michelsen P K, Moseev D, Nielsen S K, Reiter B, Stober J, Wagner D, Woskov P and the ASDEX Upgrade team 2010 J. Phys.: Conf. Ser. 227012010

[31] Freethy S J, McClements K G, Chapman S C, Dendy R O, Lai W N, Pamela S J P, Shevchenko V F and Vann R G L 2015 Phys. Rev. Lett. 114125004

[32] Li E, Austin M E, White R B and Taylor G 2017 Phys. Plasmas 24092509

[33] Willensdorfer M, Cote T B, Griener M, Ryan D A, Strumberger E, Suttrop W, Wang N, Cavedon M, Denk S S, Dunne M, Fischer R, Galdon-Quiroga J, Ham C J, Hegna C C, Hoelzl M, Kirk A, Maraschek M, Mink F, Leuthold N, Orain F, Seliunin E P, Zohm H, the ASDEX Upgrade Team and the MST1 Team 2019 Plasma Phys. Control. Fusion 61014019 
[34] Huysmans G T A and Czarny O 2007 Nucl. Fusion 47 659-666

[35] Mink A F, Hoelzl M, Wolfrum E, Orain F, Dunne M, Lessig A, Pamela S, Manz P, Maraschek M, Huijsmans G T A, Becoulet M, Laggner F M, Cavedon M, Lackner K, Günter S, Stroth U and The ASDEX Upgrade Team 2018 Nucl. Fusion 58026011

[36] Hoelzl M, Huijsmans G T A, Orain F, Artola F J, Pamela S, Becoulet M, van Vugt D, Liu F, Futatani S, Lessig A, Wolfrum E, Mink F, Trier E, Dunne M, Viezzer E, Eich T, Vanovac B, Frassinetti L, Guenter S, Lackner K, Krebs I, ASDEX Upgrade Team and EUROfusion MST1 Team 2018 Contrib. Plasma Phys. 58 518-528

[37] Opher M, Morales G J and Leboeuf J N 2002 Phys. Rev. E 66016407

[38] Ram A K and Schultz S D 2000 Phys. Plasmas 7 4084-4094

[39] Jones B M 2002 Electron Bernstein Wave Thermal Emission and Mode Conversion in the CDX-U Spherical Torus Ph.D. thesis Princeton University Princeton URL https://search.proquest. com/docview/305550955

[40] Laqua H P 2007 Plasma Phys. Control. Fusion 49 R1-42

[41] Lopez N A and Ram A K 2018 Plasma Phys. Control. Fusion 60125012

[42] Berk H L and Book D L 1969 Phys. Fluids 12 649-661

[43] DuBois D F, Forslund D W and Williams E A 1974 Phys. Rev. Lett. 33 1013-1016

[44] Hansen S K, Nielsen S K, Salewski M, Stejner M, Stober J and the ASDEX Upgrade team 2017 Plasma Phys. Control. Fusion 59105006

[45] Meyer H for the ASDEX Upgrade Team 2019 Nucl. Fusion 59112014

[46] Nielsen S K, Michelsen P K, Hansen S K, Korsholm S B, Leipold F, Rasmussen J, Salewski M, Schubert M, Stejner M, Stober J, Wagner D and the ASDEX Upgrade team 2017 Phys. Scr. 92 024001

[47] Stejner M, Nielsen S, Jacobsen A S, Korsholm S B, Leipold F, Meo F, Michelsen P K, Moseev D, Rasmussen J, Salewski M, Schubert M, Stober J, Wagner D H and ASDEX Upgrade Team 2014 Rev. Sci. Instrum. 85093504

[48] Furtula V, Salewski M, Leipold F, Michelsen P K, Korsholm S B, Meo F, Moseev D, Nielsen S K, Stejner M and Johansen T 2012 Rev. Sci. Instrum. 83013507

[49] Stejner M, Nielsen S, Jacobsen A S, Korsholm S B, Leipold F, McDermott R M, Rasmussen J, Salewski M, Schubert M, Stober J, Wagner D H, the ASDEX Upgrade Team and the EUROfusion MST1 Team 2016 Measuring main-ion temperatures in ASDEX Upgrade using scattering of ECRH radiation Proc. 41st Int. Conf. Infrared, Millimeter, and Terahertz Waves (Copenhagen) (Piscataway: IEEE)

[50] McCarthy P J, Martin P and Schneider W 1999 The CLISTE interpretive equilibrium code Tech. Rep. IPP 5/85 Max-Planck-Institut für Plasmaphysik Garching URL http://hdl .handle.net/ 11858/00-001M-0000-0027-6025-9

[51] Fischer R, Fuchs C J, Kurzan B, Suttrop W, Wolfrum E and the ASDEX Upgrade team 2010 Fusion Sci. Technol. 58 675-684

[52] Zohm H 1996 Plasma Phys. Control. Fusion 38 105-128

[53] Mlynek A, Schramm G, Eixenberger H, Sips G, McCormick K, Zilker M, Behler K, Eheberg J and ASDEX Upgrade Team 2010 Rev. Sci. Instrum. 81033507

[54] Hansen S K, Nielsen S K, Stober J, Rasmussen J, Salewski M, Stejner M and ASDEX Upgrade Team 2019 Phys. Plasmas 26062102

[55] Leipold F, Hansen S K, Jacobsen A S, Jensen T, Jessen M, Korsholm S B, Nielsen S K, Rasmussen J, Salewski M and Stejner M 2016 Millimeter-wave receiver design for plasma diagnostics Proc. 41st Int. Conf. Infrared, Millimeter, and Terahertz Waves (Copenhagen) (Piscataway: IEEE)

[56] Godyak V A and Kuzovnikov A A 1975 Sov. J. Plasma Phys. 1 276-280

[57] Cziegler I, Terry J L, Wukitch S J, Garrett M L, Lau C and Lin Y 2012 Plasma Phys. Control. Fusion 54105019

[58] Zhang W, Feng Y, Noterdaeme J M, Bobkov V, Colas L, Coster D, Lunt T, Bilato R, Jacquot J, Ochoukov R, Van Eester D, Křivská A, Jacquet P, Guimarais L and the ASDEX Upgrade Team 
2016 Plasma Phys. Control. Fusion 58095005

[59] Viezzer E, Pütterich T, Conway G D, Dux R, Happel T, Fuchs J C, McDermott R M, Ryter F, Sieglin B, Suttrop W, Willensdorfer M, Wolfrum E and the ASDEX Upgrade Team 2013 Nucl. Fusion 53053005

[60] Johnsen H, Pécseli H L and Trulsen J 1987 Phys. Fluids 30 2239-2254

[61] White R B and Chen F F 1974 Plasma Phys. 16 565-587

[62] Mjølhus E 1987 J. Plasma Phys. 38 1-26

[63] Airy G B 1833 Philos. Mag. 2 20-30

[64] Gusakov E Z 2002 Plasma Phys. Rep. 28 580-585

[65] Stegmeir A, Coster D, Ross A, Maj O, Lackner K and Poli E 2018 Plasma Phys. Control. Fusion 60035005

[66] Happel T, Griener M, Silvagni D, Freethy S J, Hennequin P, Janky F, Manz P, Prisiazhniuk D, Ryter F, Bernert M, Brida D, Eich T, Faitsch M, Gil L, Guimarais L, Merle A, Nille D, Pinzón J, Sieglin B, Stroth U, Viezzer E, the ASDEX Upgrade Team and the EUROfusion MST1 Team 2019 Nucl. Mater. Energy 18 159-165

[67] Labit B et al. 2019 Nucl. Fusion 59086020

[68] Greenfield C M, Burrell K H, DeBoo J C, Doyle E J, Stallard B W, Synakowski E J, Fenzi C, Gohil P, Groebner R J, Lao L L, Makowski M A, McKee G R, Moyer R A, Rettig C L, Rhodes T L, Pinsker R I, Staebler G M, West W P and the DIII-D Team 2001 Phys. Rev. Lett. 86 $4544-4547$

[69] Austin M E, Wu S M, Harvey R W and Ellis R F 2006 Investigation of narrowband ECE bursts in DIII-D plasmas Proc. 14th Joint Workshop ECE and ECRH (Santorini) (Agia Paraskevi: Hellasfusion) p 34 URL https://hellasfusion.gr/images/stories/ec14/papers/34.pdf 